\title{
Fixed-Time Synchronization for Different Dimensional Complex Network Systems with Unknown Parameters via Adaptive Control
}

\author{
Yude Ji $\mathbb{D}$, Yunli Gong $\mathbb{D}$, Shan Su, and Xiaoxue Bai \\ College of Sciences, Hebei University of Science and Technology, Shijiazhuang 050018, Hebei, China \\ Correspondence should be addressed to Yude Ji; jiyude-1980@163.com and Yunli Gong; gongyunli163@163.com
}

Received 1 December 2020; Revised 30 December 2020; Accepted 5 January 2021; Published 29 January 2021

Academic Editor: Heng Liu

Copyright (c) 2021 Yude Ji et al. This is an open access article distributed under the Creative Commons Attribution License, which permits unrestricted use, distribution, and reproduction in any medium, provided the original work is properly cited.

\begin{abstract}
This article is related to the issue of fixed-time synchronization of different dimensional complex network systems with unknown parameters. Two suitable adaptive controllers and dynamic parameter estimations are proposed such that the complex network driving and response systems can be synchronized in the settling time. Based on fixed-time control theory and Lyapunov functional method, novel sufficient conditions are provided to guarantee the synchronization within the fixed times, and the settling times are explicitly evaluated, which are independent of the initial synchronization errors. Finally, a numerical example is given to illustrate the effectiveness of the proposed control algorithms.
\end{abstract}

\section{Introduction}

Complex network systems (CNSs) usually comprise a family of coupled interconnected nodes, in which each node represents a dynamical system. Over the past decades, the field of CNS [1-6] has been gaining wide attention due to its broad applications in nature and human society, such as World Wide Web, metabolic pathways, ecological networks, food webs, electrical power grids, and so on. The synchronization of CNS has been intensively investigated due to its important applications in secure communication $[7,8]$, image encryption $[9,10]$, automatic control [11], information processing [12], and other fields. Synchronization refers to a process that all the nodes seek to adjust a certain property of their motion to a common behavior as time evolves. Generally speaking, there are two typical synchronizations of CNS including inner synchronization, i.e. synchronization of all the nodes inside every network, and outer synchronization between whole complex networks simultaneously. In recent years, many synchronization patterns have been proposed to the CNS, for instance, exponential synchronization [13-15], cluster synchronization $[16,17]$, phase synchronization [18], robust synchronization [19], generalized synchronization [20], projective synchronization [21, 22], complete synchronization [23], antisynchronization [24], etc. There are several control approaches for the synchronization of CNS, including sliding mode control [25], intermittent control and impulsive control $[26,27]$, pinning adaptive control [28, 29], optimal control [30], fuzzy control [31] and so on.

In the analysis of synchronization problems, the convergence rate of the proposed controller has been considered as a practically important issue, which leads to the investigation of finite-time synchronization [32-39]. On the one hand, compared with the infinite-time synchronization, finite-time synchronization essentially requires high-precision convergence; furthermore, the states of CNS remain completely identical after some finite time, which is called the settling time. On the other hand, a critical issue of finitetime synchronization is that the settling time heavily depends upon the initial synchronization error of considered systems. Therefore, the modification of initial synchronization errors will lead to the different settling times. However, the initial conditions of many practical systems might be random, which leads to the inaccessibility of settling time. To overcome the defect of finite-time control, the concept of fixed-time stability was firstly proposed by Polyakov [40]. Different from the finite-time method, the settling time of fixed-time technique can be estimated offline. Namely, the stability of system can be stabilized in a 
predefined time by properly selecting control gains [41, 42]. Until now, many relevant results concerning fixed-time synchronization of CNS have been published in [43-48]. The fixed-time controller for delayed memristor-based recurrent neural networks is discussed in [43]. The fixed-time synchronization of CNS with nonidentical nodes and stochastic noise perturbations was discussed in [44]. Specially, regardless of the initial conditions, the settling time of fixedtime synchronization for hybrid coupled networks can be adjusted to the desired values in [45]. In addition, based on a new fast fixed-time control method and delay feedback control, the fixed-time synchronization for the delayed neural networks and delayed Cohen-Grossberg neural networks was investigated in [46, 47], respectively. The fixed-time cluster synchronization for CNS via pinning control is considered in [48]. By designing new Lyapunov function and comparison systems, the fixed-time synchronization of CNS with impulsive effects via nonchattering control was investigated in [49]. However, most of the aforementioned results just presented either were more specific to synchronization of some systems or did not analyze the effects of unknown parameters for systems.

From the practical point of view, uncertainties in the plant models may arise due to parameter variation, neglected dynamics, unmodeled dynamics, and either unknown or approximately known parameters. Therefore, it is important and necessary to study the synchronization in such systems with unknown parameters. In recent years, some results of synchronization for CNS with unknown parameters have been obtained. In [50], the adaptive synchronization was studied for the response system contains unknown uncertain nonlinearities and unknown dead zones in neural networks. In [51], based on the invariant principle of functional differential equations and parameter identification, the rigorous adaptive feedback scheme is derived to achieve synchronization of two coupled neural networks with timevarying delay. But in real application, the parameters of the CNS may be mismatched. Paper [52] proved that for the general complex networks with identification of the topological structure and unknown parameters, synchronization can be achieved by using adaptive control approach. The adaptive control, as presented in $[51,52]$, evaluates the unknown parameters to achieve synchronization of two networks. The adaptive finite-time synchronization of different dimensional coupled chaotic (or hyperchaotic) systems with unknown parameters has been discussed in $[53,54]$. In [55], the Lyapunov stability theory and the nonlinear feedback control were used to get the robust finitetime synchronization for the chaotic systems with different orders. However, this paper does not analyze the effects of finite time adaptive feedback control on unknown parameters identification of two chaotic neural networks. Recently, the generalized outer synchronization between two nondissipatively coupled CNSs with different time-varying coupling delays was investigated in [56]. Based on new concepts of finite-time passivity, two models of delayed multiweighted CNS with different dimensional nodes were presented in [57]. The finite-time synchronization problem of all parameters estimation between two different dimensional fractional-order complex dynamical networks was proposed in [58]. By using fractional adaptive laws, the fractional-order nonlinear systems with actuator faults whose parametric uncertainties and parameters are fully unknown were investigated in $[59,60]$, respectively. These works have provided important theoretical and application values than the previous model for single driving and single response system.

It is worthwhile to emphasize that all results above only considered the same dimensional CNS, while the different dimensional CNSs are very universal. Actually, we can observe that both circulatory and respiratory systems behave in synchronous way, but their models are essentially different and they have different dimensions. So, the theoretical research of synchronization for strictly different dynamical systems is very important. Although many finite-time synchronization schemes have been developed in the literature for various CNSs, to the best of the authors' knowledge, few published papers consider the fixed-time synchronization and identification of unknown parameters for the different dimensional CNSs. So, it is meaningful to consider fixed-time synchronization of all parameters' estimation for different dimensional CNSs. This paper aims to solve this challenging problem.

The remainder of this paper is organized as follows. The preliminary results and problem formulation are given in Section 2. The main results are derived in Section 3. The numerical simulation is provided to verify the results in Section 4. The conclusion is drawn in Section 5.

Throughout the paper, the following notations will be used. Let $\mathbb{R}^{n}$ denote $n$-dimension real space and $\mathbb{R}^{+}$denote 1-dimension positive real space, and $I_{n}$ is the identity matrix with $n$ dimensions. For any $x \in \mathbb{R}^{n}$, let $\|x\|=\left(x^{T} x\right)^{(1 / 2)}$, and superscript $T$ denotes the transpose of a vector or matrix. For a matrix $A \in \mathbb{R}^{n \times n}\left(\in \mathbb{R}^{n \times m}\right), A^{-1}$ denotes the inverse matrix of $A$ (the right inverse matrix of $A$ ) and $A \otimes B$ represents the Kronecker product of matrices of $A$ and $B$. For any vector $s(t)=\left(s_{1}, s_{2}, \ldots, s_{n}\right)^{T} \in \mathbb{R}^{n},|s(t)|^{\mu}=\left(\left|s_{1}\right|^{\mu}\right.$, $\left.\left|s_{2}\right|^{\mu}, \ldots,\left|s_{n}\right|^{\mu}\right)^{T}, \quad \operatorname{sign} s(t)=\left(\operatorname{sign}\left(s_{1}\right), \operatorname{sign}\left(s_{2}\right), \ldots, \operatorname{sign}\right.$ $\left.\left(s_{n}\right)\right)^{T}$, and $\operatorname{sig}^{\mu}(s(t))=\left(\operatorname{sign}\left(s_{1}\right)\left|s_{1}\right|^{\mu}, \operatorname{sign}\left(s_{2}\right)\left|s_{2}\right|^{\mu}, \ldots\right.$, $\left.\operatorname{sign}\left(s_{n}\right)\left|s_{n}\right|^{\mu}\right)^{T}$, where $\operatorname{sign}(\cdot)$ is the standard sign function.

\section{Preliminary Results and Problem Formulation}

In order to facilitate the synchronization control of different dimensional CNSs with unknown parameters, some definitions and lemmas are presented in the section.

For the following driving system:

$$
\dot{x}=F(x(t)), \quad x(0)=x_{0},
$$

where $x(t) \in \mathbb{R}^{n}$ represents the system state, $F: \mathbb{R}^{n} \longrightarrow \mathbb{R}^{n}$ is a continuous function on an open neighbourhood $D \subseteq \mathbb{R}^{n}$ of the origin with $F(0)=0$.

The controlled response system is given by

$$
\dot{y}=G(y(t))+u(t)
$$


where $y(t) \in \mathbb{R}^{m}$ represents the system state, $u(t) \in \mathbb{R}^{m}$ represents the control input, and $G: \mathbb{R}^{m} \longrightarrow \mathbb{R}^{m}$ is a continuous function.

Remark 1. When $n=m$, the driving and response systems (1) and (2) have the same dimension. There are a lot of relevant works about the problem of synchronization. When $n \neq m$, the generalized synchronization of systems (1) and (2) with different dimensional is studied in [53-55]. There are few results about the finite-time and fixed-time synchronization of systems (1) and (2) with different dimensions. Therefore, it is essential to give some theoretical results. In addition, it should be noticed that lots of well-known chaotic systems could be written in the form of (1) and (2), such as, Lorenz system, Chen system, Rössler system, Duffing system, hyperchaotic Lorenz system, and hyperchaotic Rössler system.

The definition of fixed-time generalized synchronization is given as follows.

Definition 1. Systems (1) and (2) are said to be in finite-time generalized synchronization by adding a suitable designed controller to response system (2), if there exist two continuously differentiable functions $\phi: \mathbb{R}^{n} \longrightarrow \mathbb{R}^{r}, \varphi$ : $\mathbb{R}^{m} \longrightarrow \mathbb{R}^{r}$ and there exists a number $\widehat{T}(e(0)) \geq 0$ such that

$$
\begin{aligned}
\lim _{t \longrightarrow \widehat{T}(e(0))}\|e(t)\| & =0, \\
\|e(t)\| & =0, \quad \text { for all } t>\widehat{T}(e(0)),
\end{aligned}
$$

where $e(t)=\phi(x)-\varphi(y) \in \mathbb{R}^{r}$ represents the synchronization error of different dimensional systems (1) and (2) and $\|\cdot\|$ denotes the Euclidean norm.

The time

$$
T(e(0))=\inf \{\widehat{T}(e(0)) \geq 0:\|e(t)\|=0, \text { for all } t>\widehat{T}(e(0))\},
$$

is called the settling time of synchronization, which is dependent of the initial value $e(0)$. Furthermore, if there exists a fixed-time $T \geq 0$ such that $T(e(0)) \leq T$ for any $e(0) \in \mathbb{R}^{r}$, systems (1) and (2) are said to be in fixed-time generalized synchronization.

Remark 2. In Definition 1, the functions $\phi$ and $\varphi$ are two arbitrary continuously differentiable functions; according to the different form of functions $\phi$ and $\varphi$, the generalized synchronization can also be reduced to the projective synchronization, so the research about synchronization has more extensive meaning than previous results. If $m=n, \phi(x)=x, \varphi(y)=\beta y$, where $\beta$ is a scalar, then the generalized synchronization will be reduced to the projective synchronization and $\beta$ denotes the projective coefficient. Especially, if $\beta=1$, then the generalized synchronization will be reduced to the complete synchronization; if $\beta=-1$, then the generalized synchronization will be reduced to the projective antisynchronization.

Remark 3. If $m=n, \phi(x) \equiv 0, \varphi(y)=y$, the equation in Definition 1 is reduced to the following form:

$$
\begin{aligned}
\lim _{t \longrightarrow \widehat{T}(y(0))}\|y(t)\| & =0, \\
\|y(t)\| & =0, \quad \text { for all } t>\widehat{T}(y(0)) .
\end{aligned}
$$

In this case, system (2) is said to be globally finite-time asymptotically stabilized to the origin.

The following lemmas are useful for our main results.

Lemma 1 (see [61]). Let $a_{1}, a_{2}, \ldots, a_{N} \geq 0$ and $0<p \leq 1, q>1$. Then, the following two inequalities hold:

$$
\begin{aligned}
& \sum_{i=1}^{N} \alpha_{i}^{p} \geq\left(\sum_{i=1}^{N} \alpha_{i}\right)^{p}, \\
& \sum_{i=1}^{N} \alpha_{i}^{q} \geq N^{1-q}\left(\sum_{i=1}^{N} \alpha_{i}\right)^{q} .
\end{aligned}
$$

Lemma 2 (see [62]). Consider a scalar system

$$
\begin{aligned}
\dot{y} & =-\alpha y^{(m / n)}-\beta y^{(p / q),} \\
y(0) & =y_{0},
\end{aligned}
$$

where $\alpha>0$ and $\beta>0$ and $m, n, p, q$ are positive odd integers satisfying $m>n$ and $p<q$. Then, the equilibrium of system (7) is fixed-time stable and the settling time is

$$
T=\frac{n}{\alpha(m-n)}+\frac{q}{\beta(q-p)} \text {. }
$$

In this paper, the fixed time and adaptive controller are designed to realize the synchronization between the driving network systems and response ones with unknown parameters in the settling time $T$, i.e., $\lim _{t \longrightarrow T}\|e(t)\|=0$.

\section{Main Results}

In this section, the sufficient conditions of fixed-time synchronization for two different dimensional CNSs are obtained via adaptive control methods.

We consider the following driving dynamical networks consisting of $N$ identical nodes:

$$
\dot{x}_{i}(t)=F_{i}\left(x_{i}(t)\right)+f_{i}\left(x_{i}(t)\right) \Phi_{i}+\sum_{j=1}^{N} a_{i j} x_{j}(t), \quad i=1,2, \ldots, N,
$$

where $x_{i}(t)=\left(x_{i 1}(t), x_{i 2}(t), \ldots, x_{i n}(t)\right)^{T} \in \mathbb{R}^{n}$ denotes the state vector of $i$ th node, $F_{i}: \mathbb{R}^{n} \longrightarrow \mathbb{R}^{n}, f_{i}: \mathbb{R}^{n} \longrightarrow \mathbb{R}^{n} \times \mathbb{R}^{p}$ are continuous functions, and $\Phi_{i}=\left(\Phi_{i 1}, \Phi_{i 2}, \cdots \Phi_{i p}\right)^{T} \in \mathbb{R}^{p}$ represents the vector of unknown parameters. $A=\left(a_{i j}\right) \in \mathbb{R}^{N \times N}$ denotes the weight configuration matrix. If there is a connection between nodes $i$ and $j(i \neq j)$, then $a_{i j}=a_{j i}>0$; otherwise, $a_{i j}=a_{j i}=0$. The diagonal elements of matrices $A$ are defined as

$$
a_{i i}=-\sum_{j=1, j \neq i}^{N} a_{i j}
$$


For simplicity, driving system (9) is inferred to be in the following form:

$$
\dot{x}(t)=F(x(t))+f(x(t)) \Phi+\left(A \otimes I_{n}\right) x(t),
$$

where $\quad x(t)=\left(x_{1}^{T}(t), x_{2}^{T}(t), \ldots, x_{N}^{T}(t)\right)^{T}, F(x(t))=\left(F_{1}^{T}\right.$ $\left.\left(x_{1}(t)\right), F_{2}^{T}\left(x_{2}(t)\right), \ldots, F_{N}^{T}\left(x_{N}(t)\right)\right)^{T}, \quad f(x(t))=\operatorname{diag}\left\{f_{1}\right.$ $\left.\left(x_{1}(t)\right), f_{2}\left(x_{2}(t)\right), \ldots, f \quad N\left(x_{N}(t)\right)\right\}, \Phi=\left(\Phi_{1}^{T}, \Phi_{2}^{T}, \ldots\right.$, $\left.\Phi_{N}^{T}\right)^{T}$. follows:

Correspondingly, the response system can be given as

$$
\begin{aligned}
\dot{y}_{i}(t)= & G_{i}\left(y_{i}(t)\right)+g_{i}\left(y_{i}(t)\right) \Psi_{i} \\
& +\sum_{j=1}^{N} a_{i j} y_{j}(t)+u_{i}(t), \quad i=1,2, \ldots, N .
\end{aligned}
$$

Similarly, response system (12) is inferred to be in the following form:

$$
\dot{y}(t)=G(y(t))+g(y(t)) \Psi+\left(A \otimes I_{m}\right) y(t)+u(t),
$$

where $y(t)=\left(y_{1}^{T}(t), y_{2}^{T}(t), \ldots, y_{N}^{T}(t)\right)^{T}, y_{i}(t)=\left(y_{i 1}(t)\right.$, $\left.y_{i 2}(t), \ldots, y_{i m}(t)\right)^{T} \in \mathbb{R}^{m}$ denotes the response state vector of the $i$ th node, $G(y(t))=\left(G_{1}^{T}\left(y_{1}(t)\right), G_{2}^{T}\left(y_{2}(t)\right), \ldots\right.$, $\left.G_{N}^{T}\left(y_{N}(t)\right)\right)^{T}, G_{i}: \mathbb{R}^{m} \longrightarrow \mathbb{R}^{m}, g(y(t))=\operatorname{diag}\left\{g_{1}\left(y_{1}(t)\right)\right.$, $\left.g_{2}\left(y_{2}(t)\right), \ldots, g_{N}\left(y_{N}(t)\right)\right\}, g_{i}: \mathbb{R}^{m} \longrightarrow \mathbb{R}^{m} \times \mathbb{R}^{q}$ are continuous functions, $\Psi=\left(\Psi_{1}^{T}, \Psi_{2}^{T}, \ldots, \Psi_{N}^{T}\right)^{T}, \Psi_{i}=$ $\left(\Psi_{i 1}, \Psi_{i 2}, \cdots \Psi_{i q}\right)^{T} \in \mathbb{R}^{q}$ represent the vector of unknown parameters, and $u(t)=\left(u_{1}^{T}(t), u_{2}^{T}(t), \ldots, u_{N}^{T}(t)\right)^{T}$, $u_{i}(t) \in \mathbb{R}^{m}$ denotes the control input of the $i$ th node.

Consider the driving system (9) and response system (12) with unknown parameters; we define the synchronization error as $e(t)=\left(e_{1}^{T}(t), e_{2}^{T}(t), \ldots, e_{N}^{T}(t)\right)^{T}, e_{i}(t)=$ $\phi\left(x_{i}(t)\right)-\varphi\left(y_{i}(t)\right), \quad$ where $\phi=\left(\phi_{1}, \phi_{1}, \ldots, \phi_{r}\right)^{T}$ : $\mathbb{R}^{n} \longrightarrow \mathbb{R}^{r}$ and $\varphi=\left(\varphi_{1}, \varphi_{1}, \ldots, \varphi_{r}\right)^{T}: \mathbb{R}^{m} \longrightarrow \mathbb{R}^{r}$ are both continuously differentiable functions; then, it is well known that the fixed-time synchronization problem between driving system (9) and response system (12) can be transformed to the equivalent problem of the fixed-time stabilization for the following error system:

$$
\begin{aligned}
\dot{e}_{i}(t)= & J_{\phi}\left(x_{i}\right)\left(F_{i}\left(x_{i}\right)+\sum_{j=1}^{N} a_{i j} x_{j}(t)+f_{i}\left(x_{i}\right) \Phi_{i}\right) \\
& -J_{\varphi}\left(y_{i}\right)\left(G_{i}\left(y_{i}\right)+\sum_{j=1}^{N} a_{i j} y_{j}(t)+g_{i}\left(y_{i}\right) \Psi_{i}+u_{i}(t)\right),
\end{aligned}
$$

where

$$
J_{\phi}\left(x_{i}\right)=\left(\begin{array}{cccc}
\frac{\partial \phi_{1}\left(x_{i}\right)}{\partial x_{i 1}} & \frac{\partial \phi_{1}\left(x_{i}\right)}{\partial x_{i 2}} & \cdots & \frac{\partial \phi_{1}\left(x_{i}\right)}{\partial x_{\text {in }}} \\
\frac{\partial \phi_{2}\left(x_{i}\right)}{\partial x_{i 1}} & \frac{\partial \phi_{2}\left(x_{i}\right)}{\partial x_{i 2}} & \cdots & \frac{\partial \phi_{2}\left(x_{i}\right)}{\partial x_{\text {in }}} \\
\vdots & \vdots & \ddots & \vdots \\
\frac{\partial \phi_{r}\left(x_{i}\right)}{\partial x_{i 1}} & \frac{\partial \phi_{r}\left(x_{i}\right)}{\partial x_{i 2}} & \cdots & \frac{\partial \phi_{r}\left(x_{i}\right)}{\partial x_{i n}}
\end{array}\right),
$$

denote the Jacobin matrices of functions $\phi\left(x_{i}\right)$ and $\varphi\left(y_{i}\right)$, respectively.

For simplicity, error system (14) is inferred to be in the following form:

$$
\begin{aligned}
\dot{e}(t)= & J_{\phi}(x)\left(F(x(t))+\left(A \otimes I_{n}\right) x(t)+f(x(t)) \Phi\right) \\
& -J_{\varphi}(y)\left(G \left(y(x(t))+\left(A \otimes I_{m}\right) y(t)\right.\right. \\
& +g(y(x(t)) \Psi+u(t)),
\end{aligned}
$$

where $J_{\phi}(x)=\operatorname{diag}\left\{J_{\phi}\left(x_{1}\right), J_{\phi}\left(x_{2}\right), \ldots, J_{\phi}\left(x_{N}\right)\right\} \quad$ and $J_{\varphi}(y)=\operatorname{diag}\left\{J_{\varphi}\left(y_{1}\right), J_{\varphi}\left(y_{2}\right), \ldots, J_{\varphi}\left(y_{N}\right)\right\}$.

Assumption 1. Let $r \leq \min \{m, n\}$ and matrix $J_{\varphi}$ be row full rank.

Now, two novel fixed-time synchronization control methodologies are given in the following two theorems.

Theorem 1. Under Assumption 1, for error system (14) of driving system (9) and response system (12) with unknown parameters, the controller $u(t)$ is designed as 


$$
\begin{aligned}
u_{i}(t)= & -G_{i}\left(y_{i}\right)-\sum_{j=1}^{N} a_{i j} y_{j}(t)-g_{i}\left(y_{i}\right) \widehat{\Psi}_{i} \\
& +J_{\varphi}^{-1}\left(y_{i}\right)\left(J_{\phi}\left(x_{i}\right)\left(F_{i}\left(x_{i}\right)+\sum_{j=1}^{N} a_{i j} x_{j}(t)+f_{i}\left(x_{i}\right) \widehat{\Phi}_{i}\right)\right. \\
& \left.+\alpha_{1} \cdot \operatorname{sig}^{\left(m_{1} / n_{1}\right)}\left(e_{i}\right)+\beta_{1} \cdot \operatorname{sig}\left(p_{1} / q_{1}\right)\left(e_{i}\right)\right),
\end{aligned}
$$

where $\widehat{\Phi}_{i}, \widehat{\Psi}_{i}$ denote the estimations of $\Phi_{i}$ and $\Psi_{i}$, $\alpha_{1}>0, \beta_{1}>0$ and $m_{1}, n_{1}, p_{1}, q_{1}$ are all positive odd integers satisfying $m_{1}>n_{1}, p_{1}<q_{1}$. And the following adaptive update laws are proposed to estimate the unknown parameters $\widehat{\Phi}_{i}$ and $\widehat{\Psi}_{i}$ :

$\dot{\widehat{\Phi}}_{i}=\left(J_{\phi}\left(x_{i}\right) f_{i}\left(x_{i}\right)\right)^{T} e_{i}(t)-\alpha_{1} \cdot \operatorname{sig}^{\left(m_{1} / n_{1}\right)}\left(\widetilde{\Phi}_{i}\right)-\beta_{1} \cdot \operatorname{sig}\left(p_{1} / q_{1}\right)\left(\widetilde{\Phi}_{i}\right)$,

$$
\begin{aligned}
\dot{\hat{\Psi}}_{i}= & -\left(J_{\varphi}\left(y_{i}\right) g_{i}\left(y_{i}\right)\right)^{T} e_{i}(t)-\alpha_{1} \cdot \operatorname{sig}^{\left(m_{1} / n_{1}\right)}\left(\widetilde{\Psi}_{i}\right)-\beta_{1} \\
& \cdot \operatorname{sig}\left(p_{1} / q_{1}\right)\left(\widetilde{\Psi}_{i}\right),
\end{aligned}
$$

where $\widetilde{\Phi}_{i}=\widehat{\Phi}_{i}-\Phi_{i}, \widetilde{\Psi}_{i}=\widehat{\Psi}_{i}-\Psi_{i}$. Then, systems (9) and (12) are fixed-time synchronized with the setting time $\left.T_{1}=\left(1 /\left(2^{\left(\left(m_{1}+n_{1}\right) /\right.} 2 n_{1}\right) \alpha_{1} N_{1}^{\left(\left(n_{1}-m_{1}\right) / 2 n_{1}\right)}\right)\right)\left(2 n_{1} /\left(m_{1}-n_{1}\right)\right)+$ $\left(1 /\left(2^{\left(\left(p_{1}+q_{1}\right) / 2 q_{1}\right)} \beta_{1}\right)\right)\left(2 q_{1} /\left(q_{1}-p_{1}\right)\right)$, where $N_{1}=N(r+p$ $+q)$.

Proof. Substituting controller (17) into the error system (14) yields

$$
\begin{aligned}
\dot{e}_{i}(t)= & -J_{\phi}\left(x_{i}\right) f_{i}\left(x_{i}\right) \widetilde{\Phi}_{i}+J_{\varphi}\left(y_{i}\right) g_{i}\left(y_{i}\right) \widetilde{\Psi}_{i}-\alpha_{1} \\
& \cdot \operatorname{sig}^{\left(m_{1} / n_{1}\right)}\left(e_{i}\right)-\beta_{1} \cdot \operatorname{sig}^{\left(p_{1} / q_{1}\right)}\left(e_{i}\right) .
\end{aligned}
$$

Consider the following Lyapunov function:

$$
V(t)=\frac{1}{2} \sum_{i=1}^{N} e_{i}^{T}(t) e_{i}(t)+\frac{1}{2} \sum_{i=1}^{N} \widetilde{\Phi}_{i}^{T} \widetilde{\Phi}_{i}+\frac{1}{2} \sum_{i=1}^{N} \widetilde{\Psi}_{i}^{T} \widetilde{\Psi}_{i} .
$$

Then, the time derivative of $V(t)$ along (20) with the adaptive tuning laws (18) and (19) can be calculated as

$$
\begin{aligned}
& \dot{V}(t)=\sum_{i=1}^{N} e_{i}^{T}(t) \dot{e}_{i}(t)+\sum_{i=1}^{N} \widetilde{\Phi}_{i}^{T} \dot{\widetilde{\Phi}}_{i}+\sum_{i=1}^{N} \widetilde{\Psi}_{i}^{T} \dot{\tilde{\Psi}}_{i} \\
& =\sum_{i=1}^{N} e_{i}^{T}(t)\left(-J_{\phi}\left(x_{i}\right) f_{i}\left(x_{i}\right) \widetilde{\Phi}_{i}+J_{\varphi}\left(y_{i}\right) g_{i}\left(y_{i}\right) \widetilde{\Psi}_{i}-\alpha_{1} \cdot \operatorname{sig}\left(m_{1} / n_{1}\right)\left(e_{i}\right)-\beta_{1} \cdot \operatorname{sig}\left(p_{1} / q_{1}\right)\left(e_{i}\right)\right) \\
& +\sum_{i=1}^{N} \widetilde{\Phi}_{i}^{T}\left(\left(J_{\phi}\left(x_{i}\right) f_{i}\left(x_{i}\right)\right)^{T} e_{i}(t)-\alpha_{1} \cdot \operatorname{sig}^{\left(m_{1} / n_{1}\right)}\left(\widetilde{\Phi}_{i}\right)-\beta_{1} \cdot \operatorname{sig}^{\left(p_{1} / q_{1}\right)}\left(\widetilde{\Phi}_{i}\right)\right) \\
& +\sum_{i=1}^{N} \widetilde{\Psi}_{i}^{T}\left(-\left(J_{\varphi}\left(y_{i}\right) g_{i}\left(y_{i}\right)\right)^{T} e_{i}(t)-\alpha_{1} \cdot \operatorname{sig}^{\left(m_{1} / n_{1}\right)}\left(\widetilde{\Psi}_{i}\right)-\beta_{1} \cdot \operatorname{sig}^{\left(p_{1} / q_{1}\right)}\left(\widetilde{\Psi}_{i}\right)\right) \\
& =-\alpha_{1} \sum_{i=1}^{N} e_{i}^{T}(t) \cdot \operatorname{sig}\left(m_{1} / n_{1}\right)\left(e_{i}\right)-\alpha_{1} \sum_{i=1}^{N} \widetilde{\Phi}_{i}^{T} \cdot \operatorname{sig}^{\left(m_{1} / n_{1}\right)}\left(\widetilde{\Phi}_{i}\right)-\alpha_{1} \sum_{i=1}^{N} \widetilde{\Psi}_{i}^{T} \cdot \operatorname{sig}^{\left(m_{1} / n_{1}\right)}\left(\widetilde{\Psi}_{i}\right) \\
& -\beta_{1} \sum_{i=1}^{N} e_{i}^{T}(t) \cdot \operatorname{sig}\left(p_{1} / q_{1}\right)\left(e_{i}\right)-\beta_{1} \sum_{i=1}^{N} \widetilde{\Phi}_{i}^{T} \cdot \operatorname{sig}\left(p_{1} / q_{1}\right)\left(\widetilde{\Phi}_{i}\right)-\beta_{1} \sum_{i=1}^{N} \widetilde{\Psi}_{i}^{T} \cdot \operatorname{sig}\left(p_{1} / q_{1}\right)\left(\widetilde{\Psi}_{i}\right) \\
& =-\alpha_{1} \sum_{i=1}^{N} \sum_{j=1}^{r}\left|e_{i j}(t)\right|^{\left(\left(m_{1}+n_{1}\right) / n_{1}\right)}-\alpha_{1} \sum_{i=1}^{N} \sum_{j=1}^{p}\left|\widetilde{\Phi}_{i j}\right|^{\left(\left(m_{1}+n_{1}\right) / n_{1}\right)}-\alpha_{1} \sum_{i=1}^{N} \sum_{j=1}^{q}\left|\widetilde{\Psi}_{i j}\right|^{\left(\left(m_{1}+n_{1}\right) / n_{1}\right)} \\
& -\beta_{1} \sum_{i=1}^{N} \sum_{j=1}^{r}\left|e_{i j}(t)\right|^{\left(\left(p_{1}+q_{1}\right) / q_{1}\right)}-\beta_{1} \sum_{i=1}^{N} \sum_{j=1}^{p}\left|\widetilde{\Phi}_{i j}\right|^{\left(\left(p_{1}+q_{1}\right) / q_{1}\right)}-\beta_{1} \sum_{i=1}^{N} \sum_{j=1}^{q}\left|\widetilde{\Psi}_{i j}\right|^{\left(\left(p_{1}+q_{1}\right) / q_{1}\right)} \text {. }
\end{aligned}
$$

Applying the Lemma 1 yields 


$$
\begin{aligned}
& \dot{V}(t) \leq-2^{\left(\left(m_{1}+n_{1}\right) / 2 n_{1}\right)} \alpha_{1} N_{1}^{\left(\left(n_{1}-m_{1}\right) / 2 n_{1}\right)}\left(\frac{1}{2} \sum_{i=1}^{N} \sum_{j=1}^{r}\left(e_{i j}(t)\right)^{2}+\frac{1}{2} \sum_{i=1}^{N} \sum_{j=1}^{p} \widetilde{\Phi}_{i}^{2}+\frac{1}{2} \sum_{i=1}^{N} \sum_{j=1}^{q} \widetilde{\Psi}_{i}^{2}\right)^{\left(\left(m_{1}+n_{1}\right) / 2 n_{1}\right)} \\
& -2^{\left(\left(p_{1}+q_{1}\right) / 2 q_{1}\right)} \beta_{1}\left(\frac{1}{2} \sum_{i=1}^{N} \sum_{j=1}^{r}\left(e_{i j}(t)\right)^{2}+\frac{1}{2} \sum_{i=1}^{N} \sum_{j=1}^{p} \widetilde{\Phi}_{i j}^{2}+\frac{1}{2} \sum_{i=1}^{N} \sum_{j=1}^{q} \widetilde{\Psi}_{i j}^{2}\right)^{\left(\left(p_{1}+q_{1}\right) / 2 q_{1}\right)} \\
& =-2^{\left(\left(m_{1}+n_{1}\right) / 2 n_{1}\right)} \alpha_{1} N_{1}^{\left(\left(n_{1}-m_{1}\right) / 2 n_{1}\right)}\left(\frac{1}{2} \sum_{i=1}^{N} e_{i}^{T}(t) e_{i}(t)+\frac{1}{2} \sum_{i=1}^{N} \widetilde{\Phi}_{i}^{T} \widetilde{\Phi}_{i}+\frac{1}{2} \sum_{i=1}^{N} \widetilde{\Psi}_{i}^{T} \widetilde{\Psi}_{i}\right)^{\left(\left(m_{1}+n_{1}\right) / 2 n_{1}\right)} \\
& -2^{\left(\left(p_{1}+q_{1}\right) / 2 q_{1}\right)} \beta_{1}\left(\frac{1}{2} \sum_{i=1}^{N} e_{i}^{T}(t) e_{i}(t)+\frac{1}{2} \sum_{i=1}^{N} \widetilde{\Phi}_{i}^{T} \widetilde{\Phi}_{i}+\frac{1}{2} \sum_{i=1}^{N} \widetilde{\Psi}_{i}^{T} \widetilde{\Psi}_{i}\right)^{\left(\left(p_{1}+q_{1}\right) / 2 q_{1}\right)} \\
& =-2^{\left(\left(m_{1}+n_{1}\right) / 2 n_{1}\right)} \alpha_{1} N_{1}^{\left(\left(n_{1}-m_{1}\right) / 2 n_{1}\right)} V(t)^{\left(\left(m_{1}+n_{1}\right) / 2 n_{1}\right)}-2^{\left(\left(p_{1}+q_{1}\right) / 2 q_{1}\right)} \beta_{1} V(t)^{\left(\left(p_{1}+q_{1}\right) / 2 q_{1}\right)} \text {. }
\end{aligned}
$$

According to Lemma 2, error system (20) is fixed-time stable with the setting time

$$
\begin{aligned}
T_{1}= & \frac{1}{2^{\left(\left(m_{1}+n_{1}\right) / 2 n_{1}\right)} \alpha_{1} N_{1}^{\left(\left(n_{1}-m_{1}\right) / 2 n_{1}\right)}} \frac{2 n_{1}}{m_{1}-n_{1}} \\
& +\frac{1}{2^{\left(\left(p_{1}+q_{1}\right) / 2 q_{1}\right)} \beta_{1}} \frac{2 q_{1}}{q_{1}-p_{1}} .
\end{aligned}
$$

The proof is completed.

For driving system (9) and response system (12) with unknown parameters, the proposed control scheme in Theorem 1 can be extended to design a new adaptive controller.

Theorem 2. Under Assumption 1, for driving system (9) and response system (12) with unknown parameters, the controller $u(t)$ is designed as

$$
\begin{aligned}
u_{i}(t)= & -G_{i}\left(y_{i}\right)-\sum_{j=1}^{N} a_{i j} y_{j}(t)-g_{i}\left(y_{i}\right) \widehat{\Psi}_{i} \\
& +J_{\varphi}^{-1}\left(y_{i}\right)\left(J_{\phi}\left(x_{i}\right)\left(F_{i}\left(x_{i}\right)+\sum_{j=1}^{N} a_{i j} x_{j}(t)+f_{i}\left(x_{i}\right) \widehat{\Phi}_{i}\right)\right. \\
& \left.+\alpha_{2} \cdot \operatorname{sig}^{\left(m_{2} / n_{2}\right)}\left(e_{i}\right)+\beta_{2} \cdot \operatorname{sig}^{\left(p_{2} / q_{2}\right)}\left(e_{i}\right)+\gamma(t) \cdot \operatorname{sig}^{\left(p_{2} / q_{2}\right)}\left(e_{i}\right)\right),
\end{aligned}
$$

where $\widehat{\Phi}_{i}, \widehat{\Psi}_{i}$ denote the estimations of $\Phi_{i}$ and $\Psi_{i}$, $\alpha_{2}>0, \beta_{2}>0$ and $m_{2}, n_{2}, p_{2}, q_{2}$ are all positive odd integers satisfying $m_{2}>n_{2}, p_{2}<q_{2}$. The following adaptive update laws are proposed to estimate the unknown parameters $\widehat{\Phi}_{i}$ and $\widehat{\Psi}_{i}$ :

$$
\begin{aligned}
\dot{\widehat{\Phi}}_{i}= & \left(J_{\phi}\left(x_{i}\right) f_{i}\left(x_{i}\right)\right)^{T} e_{i}(t)-\alpha_{2} \cdot \operatorname{sig}^{\left(m_{2} / n_{2}\right)}\left(\widetilde{\Phi}_{i}\right)-\beta_{2} \\
& \cdot \operatorname{sig}\left(p_{2} / q_{2}\right)\left(\widetilde{\Phi}_{i}\right)-\gamma(t) \cdot \operatorname{sig}\left(p_{2} / q_{2}\right)\left(\widetilde{\Phi}_{i}\right) \\
\dot{\hat{\Psi}}_{i}= & -\left(J_{\varphi}\left(y_{i}\right) g_{i}\left(y_{i}\right)\right)^{T} e_{i}(t)-\alpha_{2} \cdot \operatorname{sig}^{\left(m_{2} / n_{2}\right)}\left(\widetilde{\Psi}_{i}\right)-\beta_{2} \\
& \cdot \operatorname{sig}^{\left(p_{2} / q_{2}\right)}\left(\widetilde{\Psi}_{i}\right)-\gamma(t) \cdot \operatorname{sig}\left(p_{2} / q_{2}\right)\left(\widetilde{\Psi}_{i}\right)
\end{aligned}
$$

where $\widetilde{\Phi}_{i}=\widehat{\Phi}_{i}-\Phi_{i}, \widetilde{\Psi}_{i}=\widehat{\Psi}_{i}-\Psi_{i}$. And the adaptive updated law $\gamma(t)$ is designed as

$$
\begin{aligned}
& \dot{\gamma}(t)=\sum_{i=1}^{N} e_{i}^{T}(t) \cdot \operatorname{sig}^{\left(p_{2} / q_{2}\right)}\left(e_{i}\right)+\sum_{i=1}^{N} \widetilde{\Phi}_{i}^{T} \cdot \operatorname{sig}^{\left(p_{2} / q_{2}\right)}\left(\widetilde{\Phi}_{i}\right) \\
& +\sum_{i=1}^{N} \widetilde{\Psi}_{i}^{T} \cdot \operatorname{sig}\left(p_{2} / q_{2}\right)\left(\widetilde{\Psi}_{i}\right) \\
& -\alpha_{2} \cdot \operatorname{sign}(\gamma(t))|\gamma(t)|^{\left(m_{2} / m_{2}\right)} \\
& -\beta_{2} \cdot \operatorname{sign}(\gamma(t))|\gamma(t)|^{\left(p_{2} / q_{2}\right)} \text {. }
\end{aligned}
$$

Then, systems (9) and (12) are fixed-time synchronized with the setting time

$$
\begin{aligned}
T_{2}= & \frac{1}{2^{\left(\left(m_{2}+n_{2}\right) / 2 n_{2}\right)} \alpha_{2} N_{2}^{\left(\left(n_{2}-m_{2}\right) / 2 n_{2}\right)}} \frac{2 n_{2}}{m_{2}-n_{2}} \\
& +\frac{1}{2^{\left(\left(p_{2}+q_{2}\right) / 2 q_{2}\right)} \beta_{2}} \frac{2 q_{2}}{q_{2}-p_{2}},
\end{aligned}
$$

where $N_{2}=N(r+p+q)+1$. 
Proof. Substituting controller (25) into error system (14) yields

$$
\begin{aligned}
\dot{e}_{i}(t)= & -J_{\phi}\left(x_{i}\right) f_{i}\left(x_{i}\right) \widetilde{\Phi}_{i}+J_{\varphi}\left(y_{i}\right) g_{i}\left(y_{i}\right) \widetilde{\Psi}_{i}-\alpha_{2} \\
& \cdot \operatorname{sig}^{\left(m_{2} / m_{2}\right)}\left(e_{i}\right)-\beta_{2} \cdot \operatorname{sig}^{\left(p_{2} / q_{2}\right)}\left(e_{i}\right)-\gamma(t) \cdot \operatorname{sig}^{\left(p_{2} / q_{2}\right)}\left(e_{i}\right) .
\end{aligned}
$$

$$
V(t)=\frac{1}{2} \sum_{i=1}^{N} e_{i}^{T}(t) e_{i}(t)+\frac{1}{2} \sum_{i=1}^{N} \widetilde{\Phi}_{i}^{T} \widetilde{\Phi}_{i}+\frac{1}{2} \sum_{i=1}^{N} \widetilde{\Psi}_{i}^{T} \widetilde{\Psi}_{i}+\frac{1}{2} \gamma^{2}(t)
$$

Then, the time derivative of $V(t)$ along (30) with the adaptive tuning laws (26)-(28) can be calculated as

Consider the following positive definite function:

$$
\begin{aligned}
& \dot{V}(t)=\sum_{i=1}^{N} e_{i}^{T}(t) \dot{e}_{i}(t)+\sum_{i=1}^{N} \Phi_{i}^{T} \dot{\widetilde{\Phi}}_{i}+\sum_{i=1}^{N} \Psi_{i}^{T} \dot{\tilde{\Psi}}_{i}+\gamma(t) \dot{\gamma}(t) \\
& =\sum_{i=1}^{N} e_{i}^{T}(t)\left(-J_{\phi}\left(x_{i}\right) f_{i}\left(x_{i}\right) \widetilde{\Phi}_{i}+J_{\varphi}\left(y_{i}\right) g_{i}\left(y_{i}\right) \widetilde{\Psi}_{i}-\alpha_{2} \cdot \operatorname{sig}^{\left(m_{2} / n_{2}\right)}\left(e_{i}\right)-\beta_{2} \cdot \operatorname{sig}^{\left(p_{2} / q_{2}\right)}\left(e_{i}\right)-\gamma(t) \cdot \operatorname{sig}^{\left(p_{2} / q_{2}\right)}\left(e_{i}\right)\right) \\
& +\sum_{i=1}^{N} \widetilde{\Phi}_{i}^{T}\left(\left(J_{\phi}\left(x_{i}\right) f_{i}\left(x_{i}\right)\right)^{T} e_{i}(t)-\alpha_{2} \cdot \operatorname{sig}\left(m_{2} / n_{2}\right)\left(\widetilde{\Phi}_{i}\right)-\beta_{2} \cdot \operatorname{sig}\left(p_{2} / q_{2}\right)\left(\widetilde{\Phi}_{i}\right)-\gamma(t) \cdot \operatorname{sig}\left(p_{2} / q_{2}\right)\left(\widetilde{\Phi}_{i}\right)\right) \\
& +\sum_{i=1}^{N} \widetilde{\Psi}_{i}^{T}\left(-\left(J_{\varphi}\left(y_{i}\right) g_{i}\left(y_{i}\right)\right)^{T} e_{i}(t)-\alpha_{2} \cdot \operatorname{sig}^{\left(m_{2} / n_{2}\right)}\left(\widetilde{\Psi}_{i}\right)-\beta_{2} \cdot \operatorname{sig}^{\left(p_{2} / q_{2}\right)}\left(\widetilde{\Psi}_{i}\right)-\gamma(t) \cdot \operatorname{sig}^{\left(p_{2} / q_{2}\right)}\left(\widetilde{\Psi}_{i}\right)\right) \\
& +\gamma(t)\left(\sum_{i=1}^{N} e_{i}^{T}(t) \cdot \operatorname{sig}\left(p_{2} / q_{2}\right)\left(e_{i}\right)+\sum_{i=1}^{N} \widetilde{\Phi}_{i}^{T} \cdot \operatorname{sig}\left(p_{2} / q_{2}\right)\left(\widetilde{\Phi}_{i}\right)+\sum_{i=1}^{N} \widetilde{\Psi}_{i}^{T} \cdot \operatorname{sig}^{\left(p_{2} / q_{2}\right)}\left(\widetilde{\Psi}_{i}\right)\right. \\
& \left.-\alpha_{2} \cdot \operatorname{sign}(\gamma(t))|\gamma(t)|^{\left(m_{2} / n_{2}\right)}-\beta_{2} \cdot \operatorname{sign}(\gamma(t))|\gamma(t)|^{\left(p_{2} / q_{2}\right)}\right) \\
& =-\alpha_{2} \sum_{i=1}^{N} e_{i}^{T}(t) \cdot \operatorname{sig}^{\left(m_{2} / n_{2}\right)}\left(e_{i}\right)-\alpha_{2} \sum_{i=1}^{N} \widetilde{\Phi}_{i}^{T} \cdot \operatorname{sig}\left(m_{2} / n_{2}\right)\left(\widetilde{\Phi}_{i}\right) \\
& -\alpha_{2} \sum_{i=1}^{N} \widetilde{\Psi}_{i}^{T} \cdot \operatorname{sig}^{\left(m_{2} / n_{2}\right)}\left(\widetilde{\Psi}_{i}\right)-\alpha_{2} \gamma(t) \cdot \operatorname{sign}(\gamma(t))|\gamma(t)|^{\left(m_{2} / n_{2}\right)} \\
& -\beta_{2} \sum_{i=1}^{N} e_{i}^{T}(t) \cdot \operatorname{sig}\left(p_{2} / q_{2}\right)\left(e_{i}\right)-\beta_{2} \sum_{i=1}^{N} \widetilde{\Phi}_{i}^{T} \cdot \operatorname{sig}\left(p_{2} / q_{2}\right)\left(\widetilde{\Phi}_{i}\right)-\beta_{2} \sum_{i=1}^{N} \widetilde{\Psi}_{i}^{T} \cdot \operatorname{sig}\left(p_{2} / q_{2}\right)\left(\widetilde{\Psi}_{i}\right) \\
& -\beta_{2} \gamma(t) \cdot \operatorname{sign}(\gamma(t))|\gamma(t)|^{\left(p_{2} / q_{2}\right)} \\
& =-\alpha_{2} \sum_{i=1}^{N} \sum_{j=1}^{r}\left|e_{i j}(t)\right|^{\left(\left(m_{2}+n_{2}\right) / n_{2}\right)}-\alpha_{2} \sum_{i=1}^{N} \sum_{j=1}^{p}\left|\widetilde{\Phi}_{i j}\right|^{\left(\left(m_{2}+n_{2}\right) / n_{2}\right)}-\alpha_{2} \sum_{i=1}^{N} \sum_{j=1}^{p}\left|\widetilde{\Psi}_{i j}\right|^{\left(\left(m_{2}+n_{2}\right) / n_{2}\right)} \\
& -\alpha_{2} \gamma(t) \cdot \operatorname{sign}(\gamma(t))|\gamma(t)|^{\left(m_{2} / m_{2}\right)}-\beta_{2} \sum_{i=1}^{N} \sum_{j=1}^{r}\left|e_{i j}(t)\right|^{\left(\left(p_{2}+q_{2}\right) / q_{2}\right)}-\beta_{2} \sum_{i=1}^{N} \sum_{j=1}^{p}\left|\widetilde{\Phi}_{i j}\right|^{\left(\left(p_{2}+q_{2}\right) / q_{2}\right)} \\
& -\beta_{2} \sum_{i=1}^{N} \sum_{j=1}^{q}\left|\widetilde{\Psi}_{i j}\right|^{\left(\left(p_{2}+q_{2}\right) / q_{2}\right)}-\beta_{2} \gamma(t) \cdot \operatorname{sign}(\gamma(t))|\gamma(t)|^{\left(p_{2} / q_{2}\right)} .
\end{aligned}
$$

Applying Lemma 1 yields 


$$
\begin{aligned}
& \dot{V}(t) \leq-2^{\left(\left(m_{2}+n_{2}\right) / 2 n_{2}\right)} \alpha_{2} N_{2}^{\left(\left(n_{2}-m_{2}\right) / 2 n_{2}\right)}\left(\frac{1}{2} \sum_{i=1}^{N} \sum_{j=1}^{r}\left(e_{i j}(t)\right)^{2}+\frac{1}{2} \sum_{i=1}^{N} \sum_{j=1}^{p} \widetilde{\Phi}_{i j}^{2}+\frac{1}{2} \sum_{i=1}^{N} \sum_{j=1}^{q} \widetilde{\Psi}_{i j}^{2}+\frac{1}{2} \gamma^{2}(t)\right)^{\left(\left(m_{2}+n_{2}\right) / 2 n_{2}\right)} \\
& -2^{\left(\left(p_{2}+q_{2}\right) / 2 q_{2}\right)} \beta_{2}\left(\frac{1}{2} \sum_{i=1}^{N} \sum_{j=1}^{r}\left(e_{i j}(t)\right)^{2}+\frac{1}{2} \sum_{i=1}^{N} \sum_{j=1}^{p} \widetilde{\Phi}_{i j}^{2}+\frac{1}{2} \sum_{i=1}^{N} \sum_{j=1}^{q} \widetilde{\Psi}_{i j}^{2}+\frac{1}{2} \gamma^{2}(t)\right)^{\left(\left(p_{2}+q_{2}\right) / 2 q_{2}\right)} \\
& =-2\left(\left(m_{2}+n_{2}\right) / 2 n_{2}\right) \alpha_{2} N_{2}^{\left(\left(n_{2}-m_{2}\right) / 2 n_{2}\right)}\left(\frac{1}{2} \sum_{i=1}^{N} e_{i}^{T}(t) e_{i}(t)+\frac{1}{2} \sum_{i=1}^{N} \widetilde{\Phi}_{i}^{T} \widetilde{\Phi}_{i}+\frac{1}{2} \sum_{i=1}^{N} \widetilde{\Psi}_{i}^{T} \widetilde{\Psi}_{i}+\frac{1}{2} \gamma^{2}(t)\right)^{\left(\left(m_{2}+n_{2}\right) / 2 n_{2}\right)} \\
& -2^{\left(\left(p_{2}+q_{2}\right) / 2 q_{2}\right)} \beta_{2}\left(\frac{1}{2} \sum_{i=1}^{N} e_{i}^{T}(t) e_{i}(t)+\frac{1}{2} \sum_{i=1}^{N} \widetilde{\Phi}_{i}^{T} \widetilde{\Phi}_{i}+\frac{1}{2} \sum_{i=1}^{N} \widetilde{\Psi}_{i}^{T} \widetilde{\Psi}_{i}+\frac{1}{2} \gamma^{2}(t)\right)^{\left(\left(p_{2}+q_{2}\right) / 2 q_{2}\right)} \\
& =-2^{\left(\left(m_{2}+n_{2}\right) / 2 n_{2}\right)} \alpha_{2} N_{2}^{\left(\left(n_{2}-m_{2}\right) / 2 n_{2}\right)} V(t)^{\left(\left(m_{2}+n_{2}\right) / 2 n_{2}\right)}-2^{\left(\left(p_{2}+q_{2}\right) / 2 q_{2}\right)} \beta_{2} V(t)^{\left(\left(p_{2}+q_{2}\right) / 2 q_{2}\right)} \text {. }
\end{aligned}
$$

According to Lemma 2, error system (30) is also fixedtime stable with the setting time

$$
\begin{aligned}
T_{2}= & \frac{1}{2^{\left(\left(m_{2}+n_{2}\right) / 2 n_{2}\right)} \alpha_{2} N_{2}^{\left(\left(n_{2}-m_{2}\right) / 2 n_{2}\right)}} \frac{2 n_{2}}{m_{2}-n_{2}} \\
& +\frac{1}{2^{\left(\left(p_{2}+q_{2}\right) / 2 q_{2}\right)} \beta_{2}} \frac{2 q_{2}}{q_{2}-p_{2}} .
\end{aligned}
$$

The proof is completed.

Remark 4. In this paper, we provide two adaptive control strategies to ensure the fixed-time synchronization of CNS. Compared with the adaptive controller (17) of Theorem 1, controller (25) of Theorem 2 includes an additional control term $\gamma(t)$; such a control strategy can guarantee the fixedtime synchronization which is easier to be achieved, but the whole settling time will be increased.

Remark 5. The proposed controllers (17) of Theorem 1 and (25) of Theorem 2 can make the driving system (9) reach the response system (12) in the predefined time.

Remark 6. Note that the dynamic behavior of node in the error system (14) is discontinuous, which is different from the continuous dynamics of the traditional networks. In this paper, by designing the novel discontinuous feedback control schemes with sig function, chattering will occur. In order to weaken the chattering effect, by using new adaptive dynamic parameter estimations, a unified control framework is designed to guarantee the fixed-time synchronization of the discontinuous network driving system (9) reach response system (12).

Remark 7. The fixed-time impulsive synchronization control for network systems is inferred as follows: for the first stage, only impulsive control is exerted to make the synchronization error converge to one prescribed region. Then, for the second stage, only fixed-time control is exerted to realize the synchronization error converge to zero. Furthermore, it is worth noting that, in many practical applications, the dynamic behavior of CNS is often unknown. Thus, it is significant to develop an effective adaptive synchronization scheme for network systems via impulsive control and fixed-time control; more theoretical and practical aspects can be explored in the future work.

\section{Numerical Simulation}

In this section, a numerical example is presented to show the effectiveness of the proposed schemes obtained in the previous section. We consider the CNS with 10 nodes and choose node dynamics following different dimensional Lorenz and Chen hyperchaotic systems: 


$$
\begin{aligned}
& \left(\begin{array}{c}
\dot{x}_{i 1}(t) \\
\dot{x}_{i 2}(t) \\
\dot{x}_{i 3}(t)
\end{array}\right)=\underbrace{\left(\begin{array}{c}
0 \\
-x_{i 2}-x_{i 1} x_{i 3} \\
x_{i 1} x_{i 2}
\end{array}\right)}_{F_{i}\left(x_{i}(t)\right)}+\underbrace{\left(\begin{array}{ccc}
x_{i 2}-x_{i 1} & 0 & 0 \\
0 & x_{i 1} & 0 \\
0 & 0 & -x_{i 3}
\end{array}\right)}_{f_{i}\left(x_{i}(t)\right)} \underbrace{\left(\begin{array}{c}
\Phi_{i 1} \\
\Phi_{i 2} \\
\Phi_{i 3}
\end{array}\right)}_{\Phi_{i}} \\
& +\left(\begin{array}{l}
\sum_{j=1}^{10} a_{i j} x_{j 1}(t) \\
\sum_{j=1}^{10} a_{i j} x_{j 2}(t) \\
\sum_{j=1}^{10} a_{i j} x_{j 3}(t)
\end{array}\right), \quad i=1,2, \ldots, 10 \\
& \left(\begin{array}{c}
\dot{y}_{i 1}(t) \\
\dot{y}_{i 2}(t) \\
\dot{y}_{i 3}(t) \\
\dot{y}_{i 4}(t)
\end{array}\right)=\underbrace{\left(\begin{array}{c}
y_{i 4} \\
-y_{i 1} y_{i 3} \\
y_{i 1} y_{i 2} \\
-y_{i 2} y_{i 3}
\end{array}\right)}_{G_{i}\left(x_{i}(t)\right)} \\
& +\underbrace{\left(\begin{array}{ccccc}
y_{i 2}-y_{i 1} & 0 & 0 & 0 & 0 \\
0 & y_{i 1} & y_{i 2} & 0 & 0 \\
0 & 0 & 0 & -y_{i 3} & 0 \\
0 & 0 & 0 & 0 & y_{i 4}
\end{array}\right)}_{g_{i}\left(x_{i}(t)\right)} \underbrace{\left(\begin{array}{c}
\Psi_{i 1} \\
\Psi_{i 2} \\
\Psi_{i 3} \\
\Psi_{i 4} \\
\Psi_{i 5}
\end{array}\right)}_{\Psi_{i}} \\
& +\left(\begin{array}{l}
\sum_{j=1}^{10} a_{i j} y_{j 1}(t) \\
\sum_{j=1}^{10} a_{i j} y_{j 2}(t) \\
\sum_{j=1}^{10} a_{i j} y_{j 3}(t) \\
\sum_{j=1}^{10} a_{i j} y_{j 4}(t) \\
\sum_{j=1}^{10} a_{i j} y_{j 5}(t)
\end{array}\right)+\underbrace{\left(\begin{array}{l}
u_{i 1}(t) \\
u_{i 2}(t) \\
u_{i 3}(t) \\
u_{i 4}(t) \\
u_{i 5}(t)
\end{array}\right)}_{u_{i}(t)}, i=1,2, \ldots, 10 .
\end{aligned}
$$

As can be seen in Figure 1, the weight configuration matrix $A$ is given by 


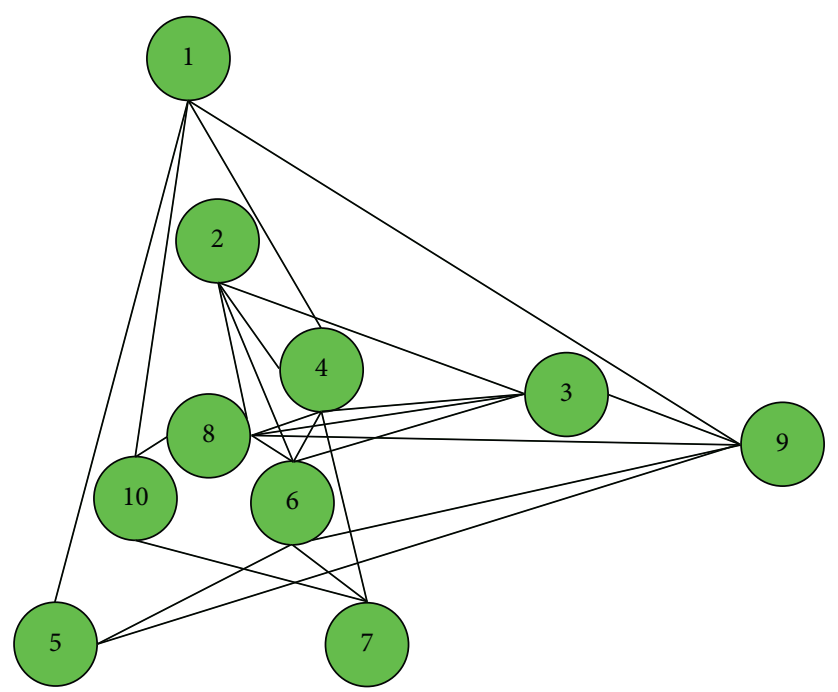

Figure 1: A simple complex dynamical network systems with ten nodes.

$$
A=\left(\begin{array}{cccccccccc}
-4 & 0 & 0 & 1 & 1 & 0 & 0 & 0 & 1 & 1 \\
0 & -4 & 1 & 1 & 0 & 1 & 0 & 1 & 0 & 0 \\
0 & 1 & -5 & 1 & 0 & 1 & 0 & 1 & 1 & 0 \\
1 & 1 & 1 & -6 & 0 & 1 & 1 & 1 & 0 & 0 \\
1 & 0 & 0 & 0 & -3 & 1 & 0 & 0 & 1 & 0 \\
0 & 1 & 1 & 1 & 1 & -7 & 1 & 1 & 1 & 0 \\
0 & 0 & 0 & 1 & 0 & 1 & -3 & 0 & 0 & 1 \\
0 & 1 & 1 & 1 & 0 & 1 & 0 & -6 & 1 & 1 \\
1 & 0 & 1 & 0 & 1 & 1 & 0 & 1 & -5 & 0 \\
1 & 0 & 0 & 0 & 0 & 0 & 1 & 1 & 0 & -3
\end{array}\right) .
$$

The associated parameters are chosen as $\Phi_{i 1}=10, \Phi_{i 2}=$ $28, \Phi_{i 3}=(8 / 3)$ and $\Psi_{i 1}=35, \Psi_{i 2}=7, \Psi_{i 3}=12, \Psi_{i 4}=3$, $\Psi_{i 5}=0.5, \quad(i=1,2, \ldots, 10)$ such that system (35) and system (36) are chaotic. According to the designing strategy of Theorems 1 and 2, select (35) as the driving system and (36) as the controlled system $(n<m)$.

Assume that $\phi\left(x_{i}\right)=\left(x_{i 1} x_{i 2}, x_{i 1}-x_{i 3}\right)^{T} \quad$ and $\varphi\left(y_{i}\right)=\left(0.5 y_{i 1}-0.5 y_{i 2}, 0.5 y_{i 3}-0.5 y_{i 4}\right)^{T}, \quad(i=1,2, \ldots$, 10). The Jacobian matrices of functions $\phi\left(x_{i}\right)$ and $\varphi\left(y_{i}\right)$ are obtained, respectively, as follows:

$$
\begin{aligned}
J_{\phi}\left(x_{i}\right) & =\left(\begin{array}{ccc}
x_{i 2} & x_{i 1} & 0 \\
1 & 0 & -1
\end{array}\right), \\
J_{\varphi}\left(y_{i}\right) & =\left(\begin{array}{cccc}
0.5 & -0.5 & 0 & 0 \\
0 & 0 & 0.5 & -0.5
\end{array}\right),
\end{aligned}
$$

and the right inverse matrix of $J_{\varphi}\left(y_{i}\right)$ is as follows:

$$
J_{\varphi}^{-1}\left(y_{i}\right)=\left(\begin{array}{cc}
1 & 0 \\
-1 & 0 \\
0 & 1 \\
0 & -1
\end{array}\right) .
$$

Compared with the controller in [53], the designed controllers can show better convergence performance in this article. Based on the controller design (27) and the error dynamics (28) in [53], we obtain

$$
\left\{\begin{array}{l}
\dot{e}_{i 1}(t)=-k \cdot \operatorname{sign}\left(e_{i 1}\right)\left|e_{i 1}\right|^{\alpha}-p \cdot e_{i 1}, \\
\dot{e}_{i 2}(t)=-k \cdot \operatorname{sign}\left(e_{i 2}\right)\left|e_{i 2}\right|^{\alpha}-p \cdot e_{i 2}
\end{array}\right.
$$

where $k=0.3, p=3, \alpha=(3 / 5)$. Moreover, the initial values of the state variable for systems (35) and (36) are given by $\left(x_{i 1}(0), x_{i 2}(0), x_{i 3}(0)\right)^{T}=(1+0.05 i,-0.05 i, 0.05 i)^{T}, \quad\left(y_{i 1}\right.$ $\left.(0), y_{i 2}(0), y_{i 3}(0), y_{i 4}(0)\right)^{T}=(1+0.05 i,-1-0.05 i,-0.05 i$, $0.05 i)^{T}, \quad(i=1,2, \ldots, 10)$, and the settling time can be estimated by $T=\left(\left(\ln (1+(p / k))\left\|e\left(t_{0}\right)\right\|^{((1-\alpha) / 2)}\right) /\right.$ $((1-\alpha) p))=2.1384$. However, the initial values of the state variable for systems (35) and (36) are chosen as $\left(x_{i 1}(0), x_{i 2}(0), x_{i 3}(0)\right)^{T}=(1+i s,-i s, i s)^{T},\left(y_{i 1}(0), y_{i 2}(0)\right.$, $\left.y_{i 3}(0) y_{i 4}(0)\right)^{T}=(1+i s,-1-i s,-i s, \quad i s)^{T}, \quad(i=1,2$, $\ldots, 10)$, where $s$ is chosen randomly on the interval $[0,0.1]$; by computation, the max settling time can be estimated by $T_{\max }=2.5903$, and the trajectories of errors are shown in Figure 2.

Based on expressions (26)-(28), by simple computations, we can obtain the adaptive updating laws in following form: 


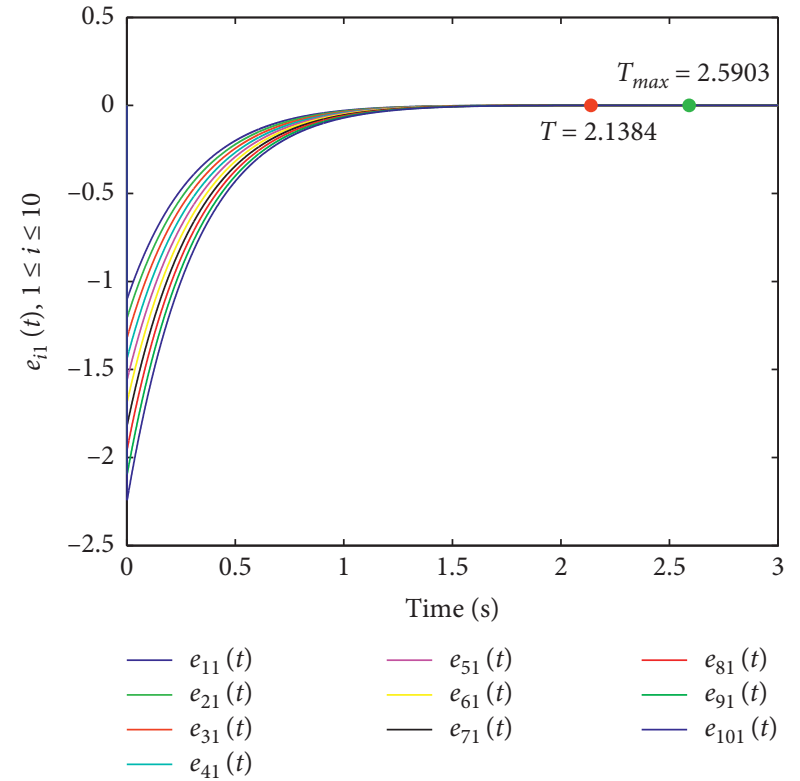

(a)

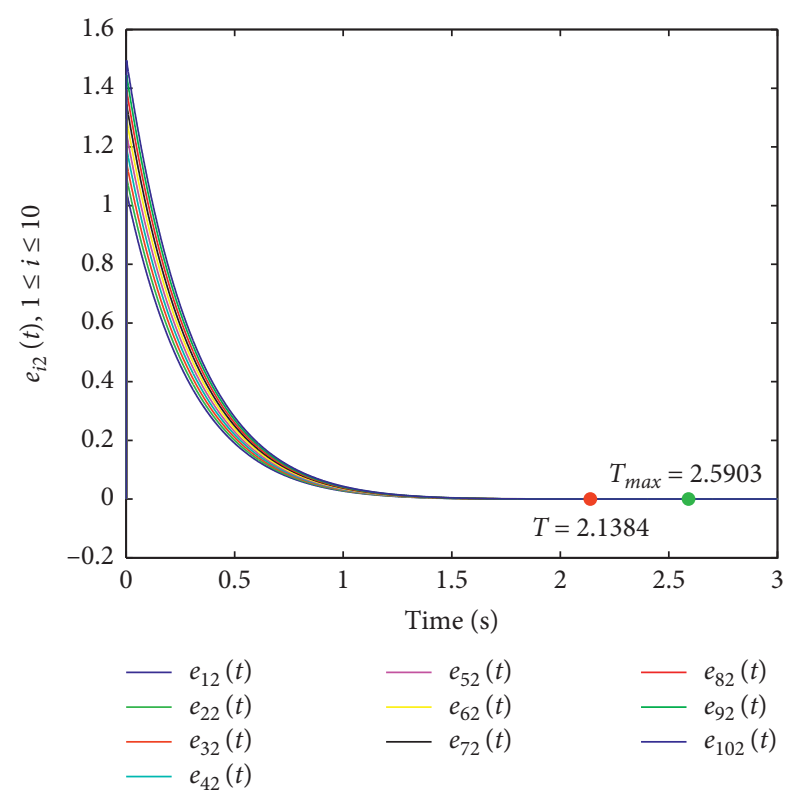

(b)

FIgURE 2: The synchronization errors between systems (35) and (36) by controller (27) in [53]. (a) $e_{i 1}(t)(i=1,2, \ldots, 10)$. (b) $e_{i 2}(t)(i=1,2, \ldots, 10)$.

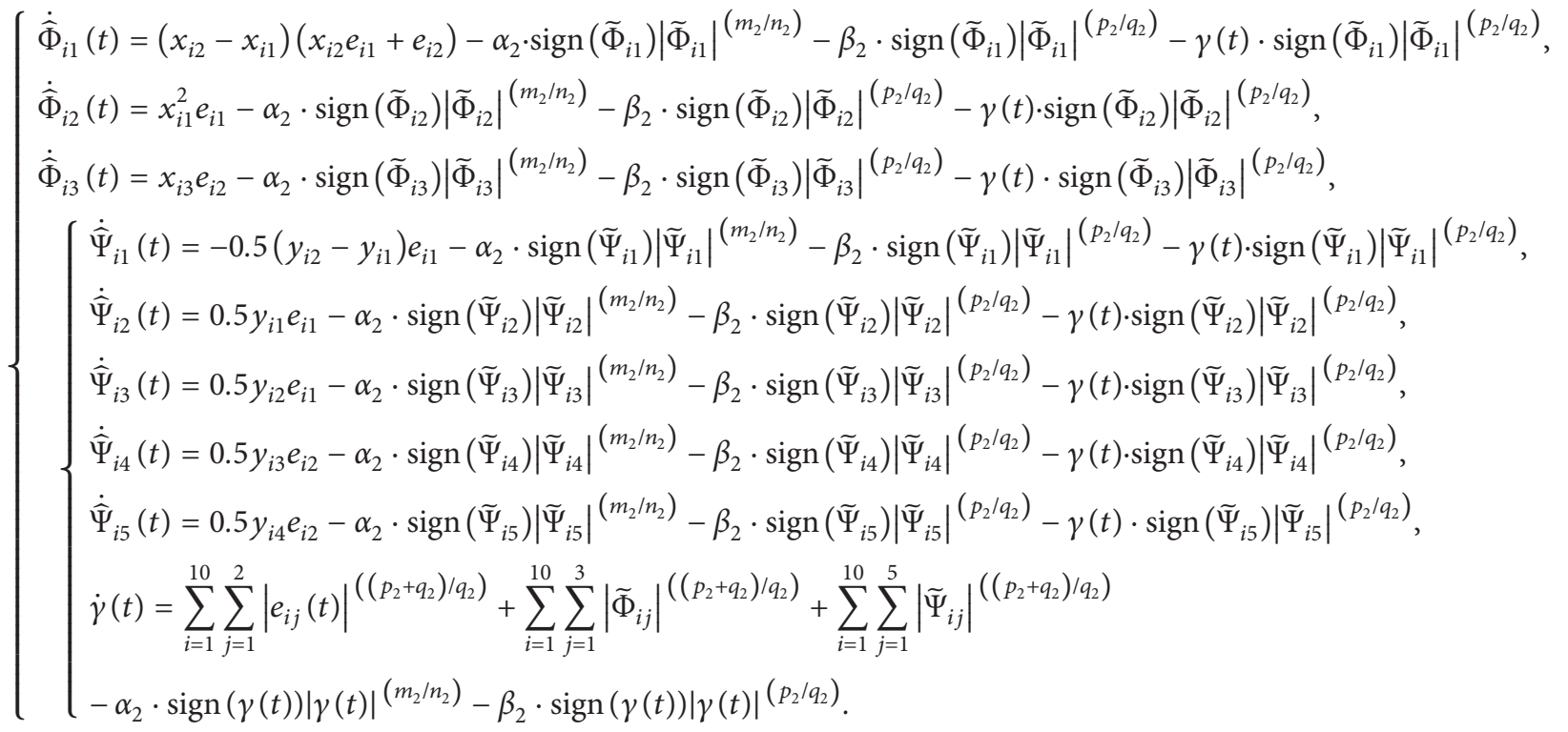




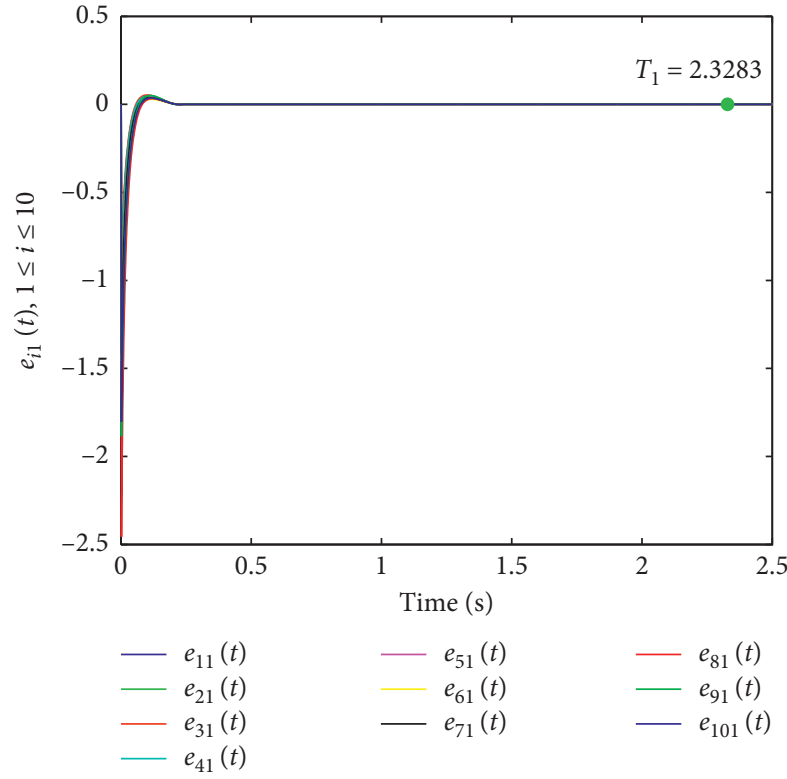

(a)

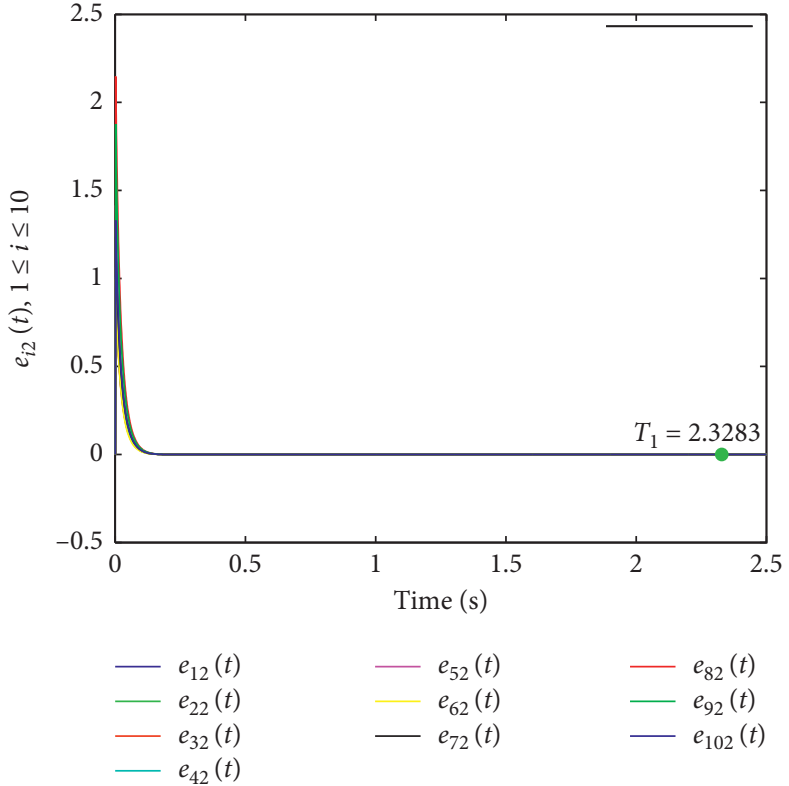

(b)

Figure 3: The synchronization errors between systems (35) and (36) by controller (17). (a) $e_{i 1}(t)(i=1,2, \ldots, 10)$. (b) $e_{i 2}(t)(i=1,2, \ldots, 10)$.

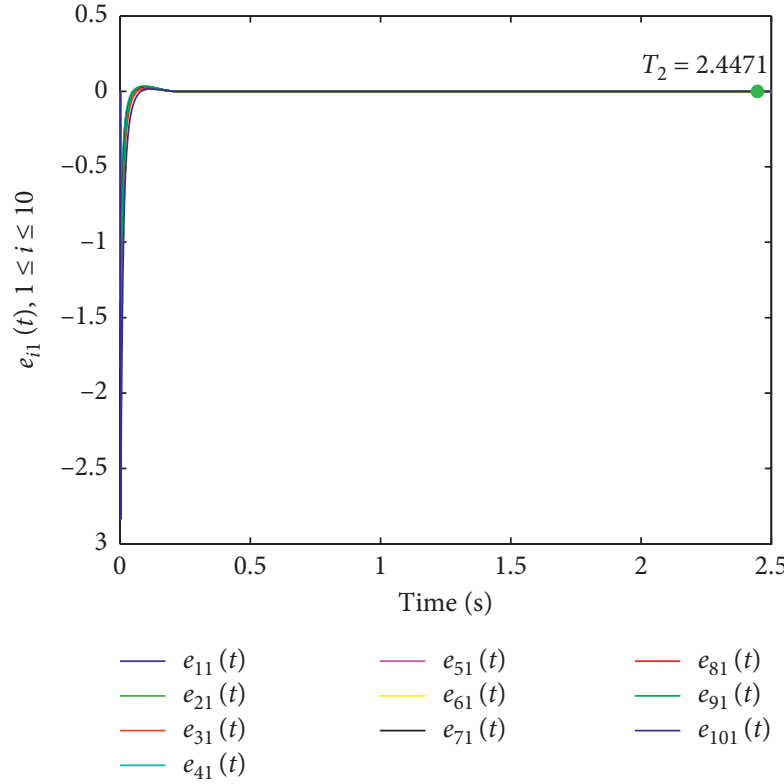

(a)

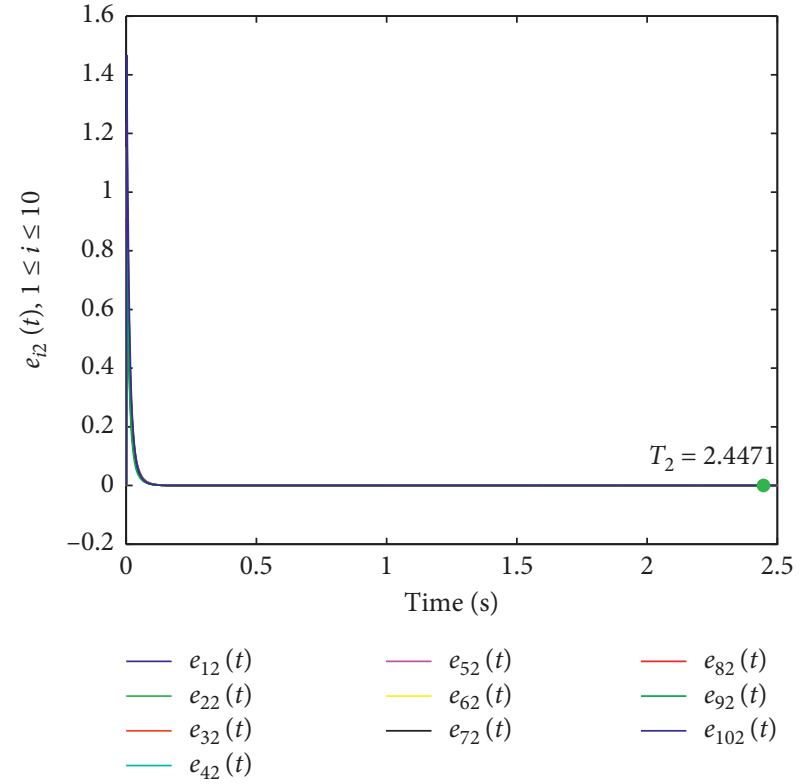

(b)

Figure 4: The synchronization errors between systems (35) and (36) by controller (25). (a) $e_{i 1}(t)(i=1,2, \ldots, 10)$. (b) $e_{i 2}(t)(i=1,2, \ldots, 10)$. 


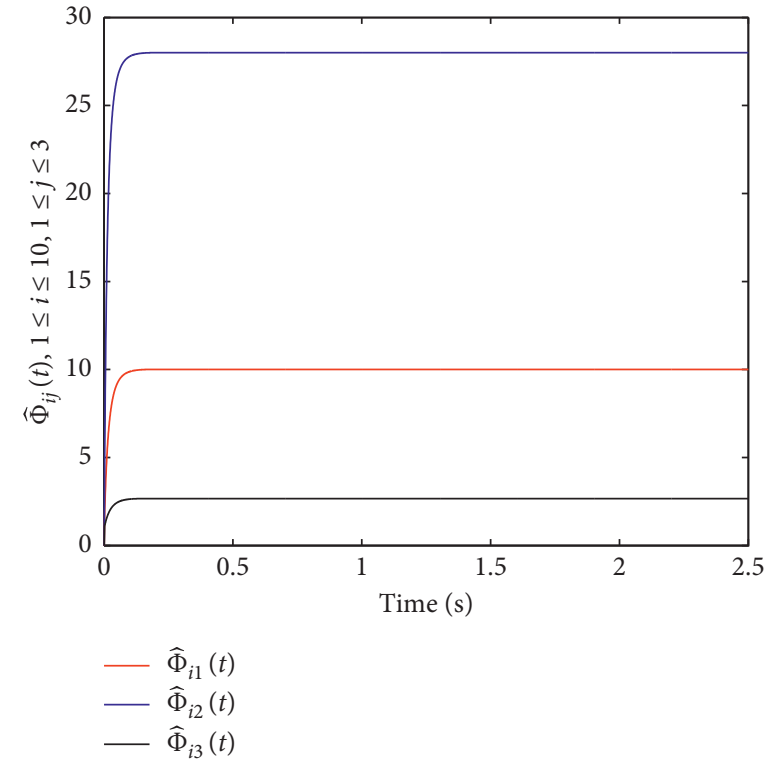

(a)

FIGURE 5: The estimations of unknown parameters for systems (b) $\widehat{\Psi}_{i}(t)(i=1,2, \ldots, 10)$.

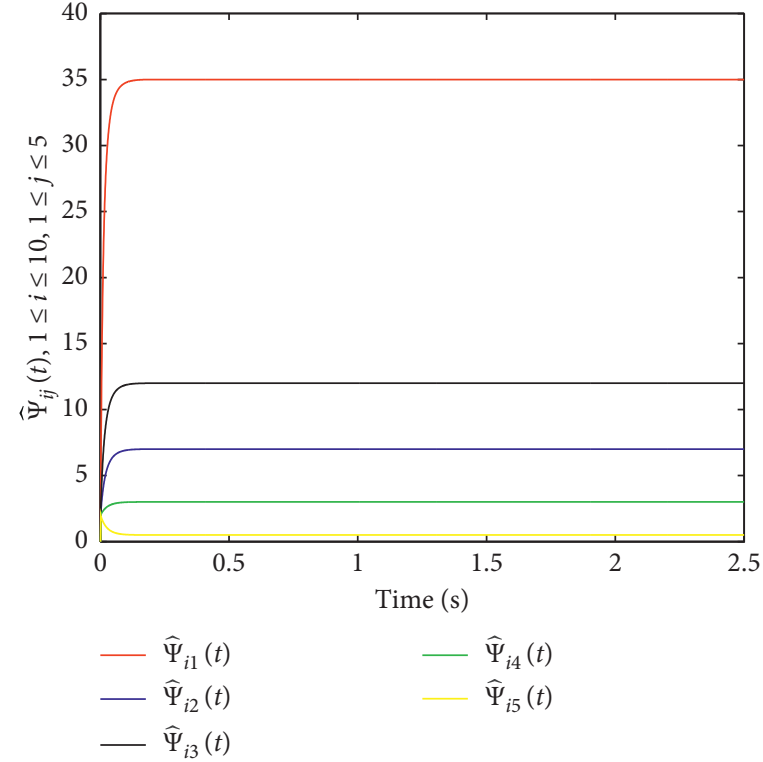

(b)

(35) and (36) by controller (17). (a) $\widehat{\Phi}_{i}(t)(i=1,2, \ldots, 10)$.
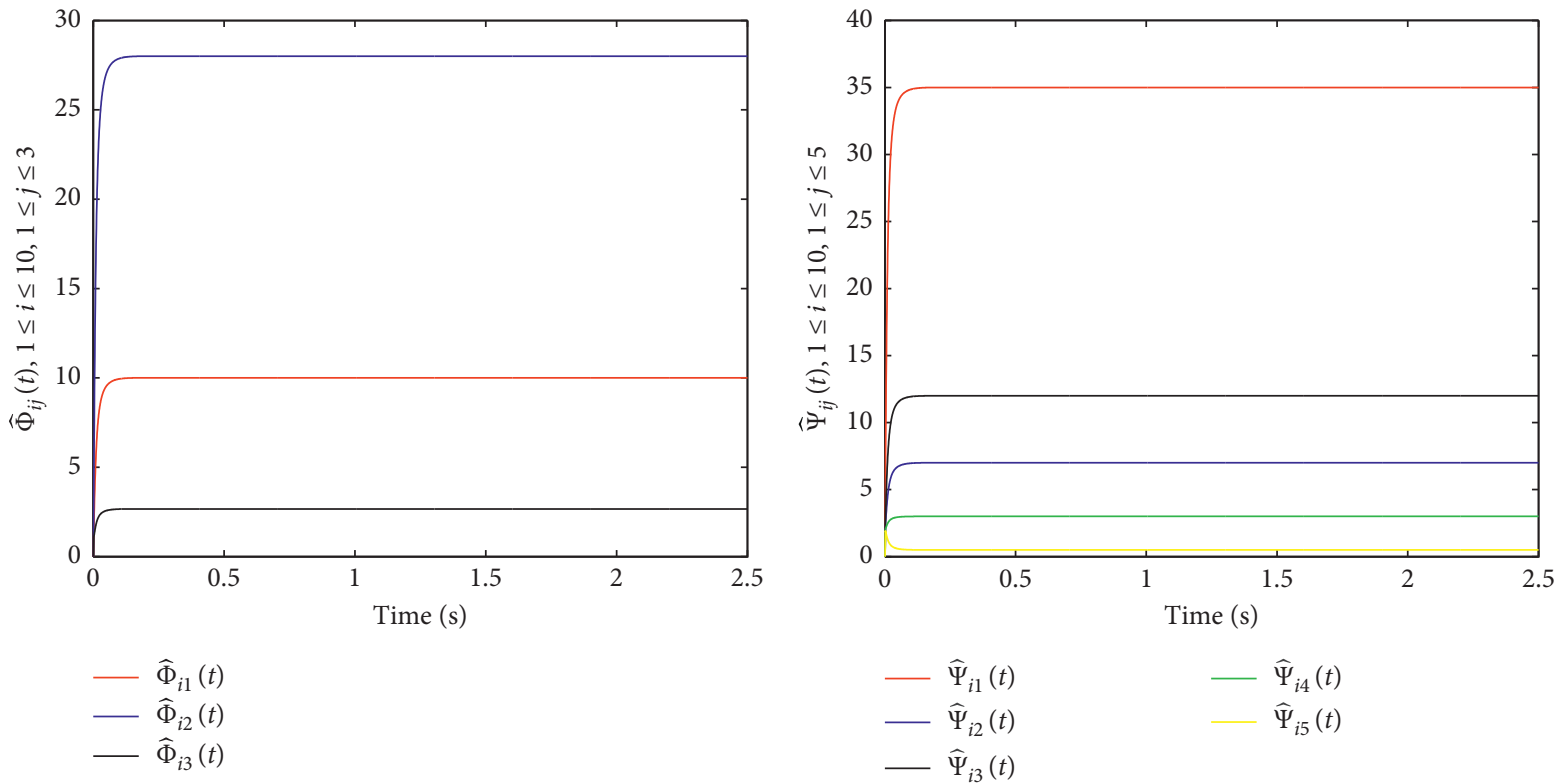

(a)

(b)

FIGURE 6: The estimations of unknown parameters for systems (35) and (36) by controller $(25) .(\mathrm{a}) \widehat{\Phi}_{i}(t)(i=1,2, \ldots, 10)$. (b) $\widehat{\Psi}_{i}(t)(i=1,2, \ldots, 10)$. 


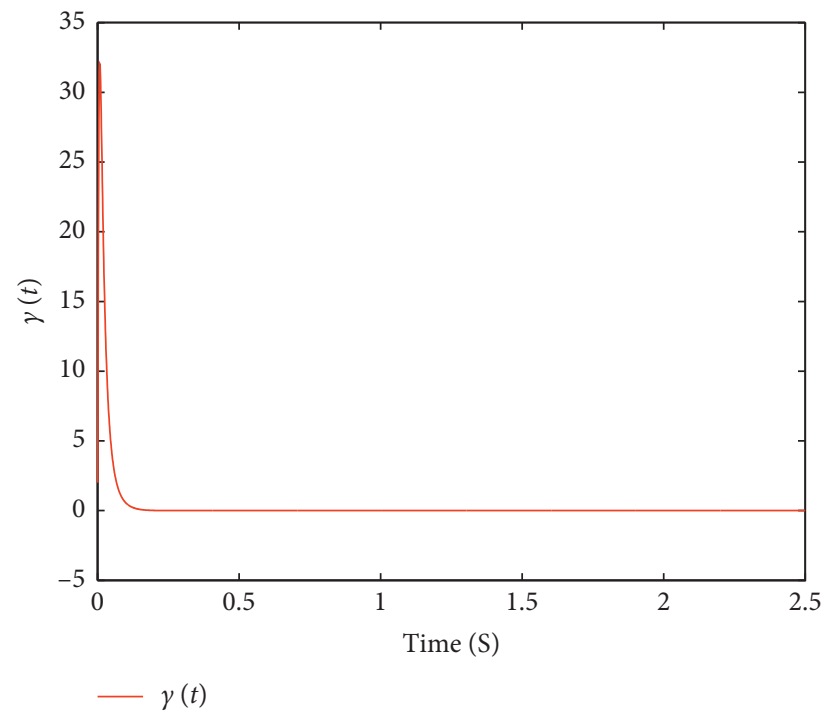

FIgURE 7: The trajectory of adaptive updated law $\gamma(t)$ by controller (25).

According to (30), the error dynamics are derived as follows:

$$
\left\{\begin{array}{l}
\dot{e}_{i 1}(t)=-x_{i 2}\left(x_{i 2}-x_{i 1}\right) \widetilde{\Phi}_{i 1}-x_{i 1}^{2} \widetilde{\Phi}_{i 2}+0.5\left(y_{i 2}-y_{i 1}\right) \widetilde{\Psi}_{i 1}-0.5 y_{i 1} \widetilde{\Psi}_{i 2}-0.5 y_{i 2} \widetilde{\Psi}_{i 3} \\
-\alpha_{2} \cdot \operatorname{sign}\left(e_{i 1}\right)\left|e_{i 1}\right|^{\left(m_{2} / m_{2}\right)}-\beta_{2} \cdot \operatorname{sign}\left(e_{i 1}\right)\left|e_{i 1}\right|^{\left(p_{2} / q_{2}\right)}-\gamma(t) \cdot \operatorname{sign}\left(e_{i 1}\right)\left|e_{i 1}\right|^{\left(p_{2} / q_{2}\right)}, \\
\dot{e}_{i 2}(t)=-\left(x_{i 2}-x_{i 1}\right) \widetilde{\Phi}_{i 1}-x_{i 3} \widetilde{\Phi}_{i 3}-0.5 y_{i 3} \widetilde{\Psi}_{i 4}-0.5 y_{i 4} \widetilde{\Psi}_{i 5} \\
-\alpha_{2} \cdot \operatorname{sign}\left(e_{i 2}\right)\left|e_{i 2}\right|^{\left(m_{2} / n_{2}\right)}-\beta_{2} \cdot \operatorname{sign}\left(e_{i 2}\right)\left|e_{i 2}\right|^{\left(p_{2} / q_{2}\right)}-\gamma(t) \cdot \operatorname{sign}\left(e_{i 2}\right)\left|e_{i 2}\right|^{\left(p_{2} / q_{2}\right)},
\end{array}\right.
$$

where the parameters are given as $m_{2}=7, n_{2}=5, p_{2}=9, q_{2}=11, \alpha_{2}=20$, and $\beta_{2}=20$. The initial values of the state variable for systems (35) and (36) are $\left(x_{i 1}(0), x_{i 2}(0), x_{i 3}(0)\right)^{T}=(1+i s,-i s, i s)^{T}, \quad\left(y_{i 1}(0)\right.$, $\left.y_{i 2}(0), y_{i 3}(0), y_{i 4}(0)\right)^{T}=(1+i s,-1-i s, \quad-i s, i s)^{T}, \quad(i=$ $1,2, \ldots, 10)$, where $s$ is chosen randomly on the interval $[0,0.1]$. Let the initial values of estimations be $\widehat{\Phi}_{i}(0)=\left(\widehat{\Phi}_{i 1}(0), \widehat{\Phi}_{i 2}(0), \widehat{\Phi}_{i 3}(0)\right)=(1,1,1)$ and $\widehat{\Psi}_{i}(0)=$ $\left(\widehat{\Psi}_{i 1}(0), \widehat{\Psi}_{i 2}(0), \widehat{\Psi}_{i 3}(0), \widehat{\Psi}_{i 4}(0), \widehat{\Psi}_{i 5}(0)\right)=(2,2,2,2,2)$, and the initial value of $\gamma(t)$ is given as $\gamma(0)=2$.

First, according to Theorem 1, systems (35) and (36) can realize fixed-time synchronization under controller (17), and settling time $T_{1}=2.3283$ by computation. The trajectories of error states $e_{i}(t)$ are shown in Figure 3. The trajectories of estimations $\widehat{\Phi}_{i}(t)$ and $\widehat{\Psi}_{i}(t)$ are depicted in Figures $4(\mathrm{a})$ and 4(b). Secondly, according to Theorem 2, systems (35) and (36) can realize fixed-time synchronization under controller (25), and the settling time $T_{2}=2.4471$ by computation. Then, the trajectories of error states $e_{i}(t)$ are shown in Figure 5. The trajectories of estimations $\widehat{\Phi}_{i}(t)$ and $\widehat{\Psi}_{i}(t)$ are depicted in Figures 6(a) and 6(b). Moreover, the trajectory of $\gamma(t)$ is illustrated in Figure 7. Finally, as shown in Figures 3 and 4 , we can find that the chaotic oscillation has been suppressed completely. Moreover, one can see from Figures 3 and 4 that systems (35) and (36) achieve synchronization within the fixed settling time $T_{1}$ and $T_{2}$, respectively.

Compared with the simulation results (see Figure 2 in the work of [53]), our adaptive law with simulation results (Figures 3 and 4 ) is faster in convergence. From Figures 5 and 6 , we can see that the estimations of unknown parameters converge to the true values rapidly. It can be concluded that with the proposed adaptive control approach, better synchronization performances are obtained compared with the previous results.

\section{Conclusions}

This paper has addressed the fixed-time synchronization of different dimensional complex network driving and response systems with unknown parameters. Two suitable controllers and adaptive update laws are established to guarantee the synchronization realized in a given fixed time, respectively. Sufficient conditions have been obtained to guarantee the fixed-time synchronization for the driving and response systems. Meanwhile, our results can be easily 
extended to the cases of projective synchronization and complete synchronization. Finally, the effectiveness of theoretical analysis has been verified by a numerical simulation.

\section{Data Availability}

All datasets generated for this study are included within the article.

\section{Conflicts of Interest}

The authors declare that there are no conflicts of interest regarding the publication of this paper.

\section{Acknowledgments}

This work was supported by the Foundation of Graduate Student Innovation Ability Training Project of Hebei Education Department (no. CXZZSS2021106) and College Students Innovative Training Program of Hebei Province (no. S202010082026).

\section{References}

[1] X. Li, Z. Wang, C. Gao, and L. Shi, "Reasoning human emotional responses from large-scale social and public media," Applied Mathematics and Computation, vol. 310, pp. 182-193, 2017.

[2] S. Tan, Y. Wang, Y. Chen, and Z. Wang, "Evolutionary dynamics of collective behavior selection and drift: flocking, collapse, and oscillation," IEEE Transactions on Cybernetics, vol. 47, no. 7, pp. 1694-1705, 2017.

[3] L. L. Jiang, W. J. Li, and Z. Wang, "Multiple effect of social influence on cooperation in interdependent network games," Scientific Reports, vol. 5, Article ID 14657, 2015.

[4] M. Yu, W. Wang, X. Luo, L. Liu, and M. Yuan, "Exponential antisynchronization control of stochastic memristive neural networks with mixed time-varying delays based on novel delay-dependent or delay-independent adaptive controller," Mathematical Problems in Engineering, vol. 2017, Article ID 8314757, 16 pages, 2017.

[5] Z. Wang, M. Jusup, R. W. Wang et al., "Onymity promotes cooperation in social dilemma experiments," Science Advances, vol. 3, no. 3, Article ID e1601444, 2017.

[6] X. L. Li, M. Jusup, Z. Wang et al., "Punishment diminishes the benefits of network reciprocity in social dilemma experiments," Proceedings of the National Academy of Sciences, vol. 115, no. 1, pp. 30-35, 2018.

[7] V. Milanović and M. E. Zaghloul, "Synchronization of chaotic neural networks and applications to communications," International Journal of Bifurcation and Chaos, vol. 6, no. 12b, pp. 2571-2585, 1996.

[8] X. Wu, C. Zhu, and H. Kan, "An improved secure communication scheme based passive synchronization of hyperchaotic complex nonlinear system," Applied Mathematics and Computation, vol. 252, pp. 201-214, 2015.

[9] S. Wen, Z. Zeng, T. Huang, Q. Meng, and W. Yao, "Lag synchronization of switched neural networks via neural activation function and applications in image encryption," IEEE Transactions on Neural Networks and Learning Systems, vol. 26, no. 7, pp. 1493-1502, 2015.

[10] C. K. Volos, I. M. Kyprianidis, and I. N. Stouboulos, "Image encryption process based on chaotic synchronization phenomena," Signal Processing, vol. 93, no. 5, pp. 1328-1340, 2013.

[11] A. Blokhin, E. Denisenko, A. Kropp, and A. Nedialkov, "The study of the synchronization process in the gearbox with automatic control," Applied Mechanics and Materials, vol. 763, pp. 71-77, 2015.

[12] Q. X. Xie, G. R. Chen, and E. M. Bollt, "Hybrid chaos synchronization and its application in information processing," Mathematical and Computer Modelling, vol. 35, no. 1-2, pp. 145-163, 2002.

[13] J.-W. Yi, Y.-W. Wang, J.-W. Xiao, and Y. Huang, "Exponential synchronization of complex dynamical networks with markovian jump parameters and stochastic delays and its application to multi-agent systems," Communications in Nonlinear Science and Numerical Simulation, vol. 18, no. 5, pp. 1175-1192, 2013.

[14] R. Zhang, D. Zeng, J. H. Park, S. Zhong, and Y. Yu, "Novel discontinuous control for exponential synchronization of memristive recurrent neural networks with heterogeneous time-varying delays," Journal of the Franklin Institute, vol. 355, no. 5, pp. 2826-2848, 2018.

[15] X. Yang, J. Cao, and J. Lu, "Stochastic synchronization of complex networks with nonidentical nodes via hybrid adaptive and impulsive control," IEEE Transactions on Circuits and Systems I: Regular Papers, vol. 59, no. 2, pp. 371-384, 2012.

[16] L. Chen and J. A. Lu, "Cluster synchronization in a complex dynamical network with two nonidentical clusters," Journal of Systems Science and Complexity, vol. 21, no. 1, pp. 20-33, 2008.

[17] W. Wu, W. J. Zhou, and T. P. Chen, "Cluster synchronization of linearly coupled complex networks under pinning control," IEEE Transactions on Circuits and Systems I: Regular Papers, vol. 56, pp. 829-839, 2009.

[18] S. G. Guan, C. H. Lai, and G. W. Wei, "Phase synchronization between two essentially different chaotic systems," Physical Review E, vol. 72, pp. 16205-16211-8, 2005.

[19] S. Wang, Y. Huang, and S. Ren, "Synchronization and robust synchronization for fractional-order coupled neural networks," IEEE Access, vol. 5, pp. 12439-12448, 2017.

[20] G. Zhang, Z. Liu, and Z. Ma, "Generalized synchronization of different dimensional chaotic dynamical systems," Chaos, Solitons \& Fractals, vol. 32, no. 2, pp. 773-779, 2007.

[21] Z. Ding and Y. Shen, "Projective synchronization of nonidentical fractional-order neural networks based on sliding mode controller," Neural Networks, vol. 76, pp. 97-105, 2016.

[22] J. Yu, C. Hu, H. Jiang, and X. Fan, "Projective synchronization for fractional neural networks," Neural Networks, vol. 49, pp. 87-95, 2014.

[23] X. F. Wang and G. R. Chen, "Synchronization in scale-free dynamical networks: robustness and fragility," IEEE Transactions on Circuits and Systems I: Fundamental Theory and Applications, vol. 49, pp. 54-62, 2002.

[24] H.-L. Li, Y.-L. Jiang, and Z.-L. Wang, “Anti-synchronization and intermittent anti-synchronization of two identical hyperchaotic Chua systems via impulsive control," Nonlinear Dynamics, vol. 79, no. 2, pp. 919-925, 2015.

[25] H. Z. Hou, Q. L. Zhang, and M. Zheng, "Cluster synchronization in nonlinear complex networks under sliding mode control," Nonlinear Dynamics, vol. 83, no. 1-2, pp. 739-749, 2016.

[26] H. Zhao, L. Li, H. Peng et al., "Finite-time synchronization for multi-link complex networks via discontinuous control," Optik, vol. 138, pp. 440-454, 2017. 
[27] D. Zhang, Y. Shen, and J. Mei, "Finite-time synchronization of multi-layer nonlinear coupled complex networks via intermittent feedback control," Neurocomputing, vol. 225, pp. 129-138, 2017.

[28] B. Li, "Pinning adaptive hybrid synchronization of two general complex dynamical networks with mixed coupling," Applied Mathematical Modelling, vol. 40, no. 4, pp. 29832998, 2016.

[29] J. Mei, M. Jiang, B. Wang, and B. Long, "Finite-time parameter identification and adaptive synchronization between two chaotic neural networks," Journal of the Franklin Institute, vol. 350, no. 6, pp. 1617-1633, 2013.

[30] G. Mei, X. Wu, D. Ning, and J.-A. Lu, "Finite-time stabilization of complex dynamical networks via optimal control," Complexity, vol. 21, no. S1, pp. 417-425, 2016.

[31] Y. H. Sun, Z. N. Wei, G. Q. Sun, P. Ju, and S. D. Huang, "Robust stabilization and synchronization of nonlinear energy resource system via fuzzy control approach," International Journal of Fuzzy Systems, vol. 14, no. 2, pp. 337-343, 2012.

[32] X. Chen, J. Cao, J. H. Park, T. Huang, and J. Qiu, "Finite-time multi-switching synchronization behavior for multiple chaotic systems with network transmission mode," Journal of the Franklin Institute, vol. 355, no. 5, pp. 2892-2911, 2018.

[33] J. Mei, M. Jiang, W. Xu, and B. Wang, "Finite-time synchronization control of complex dynamical networks with time delay," Communications in Nonlinear Science and Numerical Simulation, vol. 18, no. 9, pp. 2462-2478, 2013.

[34] A. Abdurahman, H. Jiang, and Z. Teng, "Finite-time synchronization for memristor-based neural networks with timevarying delays," Neural Networks, vol. 69, pp. 20-28, 2015.

[35] T. Jing, F. Chen, and Q. Li, "Finite-time mixed outer synchronization of complex networks with time-varying delay and unknown parameters," Applied Mathematical Modelling, vol. 39, no. 23-24, pp. 7734-7743, 2015.

[36] H. Shen, J. H. Park, and Z.-G. Wu, "Finite-time synchronization control for uncertain Markov jump neural networks with input constraints," Nonlinear Dynamics, vol. 77, no. 4, pp. 1709-1720, 2014.

[37] X. Wang, J.-a. Fang, H. Mao, and A. Dai, "Finite-time global synchronization for a class of Markovian jump complex networks with partially unknown transition rates under feedback control," Nonlinear Dynamics, vol. 79, no. 1, pp. 47-61, 2015.

[38] D. Li and J. Cao, "Finite-time synchronization of coupled networks with one single time-varying delay coupling," Neurocomputing, vol. 166, pp. 265-270, 2015.

[39] Y. Xu, W. Zhou, J. A. Fang, C. Xie, and D. Tong, "Finite-time synchronization of the complex dynamical network with nonderivative and derivative coupling," Neurocomputing, vol. 173, pp. 1356-1361, 2016.

[40] A. Polyakov, "Nonlinear feedback design for fixed-time stabilization of linear control systems," IEEE Transactions on Automatic Control, vol. 57, no. 8, pp. 2106-2110, 2012.

[41] A. Polyakov, D. Efimov, and W. Perruquetti, "Finite-time and fixed-time stabilization: implicit Lyapunov function approach," Automatica, vol. 51, pp. 332-340, 2015.

[42] A. Levant, "On fixed and finite time stability in sliding mode control," Proceeedings 52nd. IEEE Conference Decision Control, pp. 4260-4265, 2013.

[43] J. D. Cao and R. X. Li, "Fixed-time synchronization of delayed memristor-based recurrent neural networks," Science China Information Sciences, vol. 60, no. 3, 32201 pages, 2017.
[44] W. Zhang, C. Li, T. Huang, and J. Huang, "Fixed-time synchronization of complex networks with nonidentical nodes and stochastic noise perturbations," Physica A: Statistical Mechanics and its Applications, vol. 492, pp. 1531-1542, 2018.

[45] C. Chen, L. Li, H. Peng, J. Kurths, and Y. Yang, "Fixed-time synchronization of hybrid coupled networks with timevarying delays," Chaos, Solitons \& Fractals, vol. 108, pp. 4956, 2018.

[46] Y. Xu, D. Meng, C. Xie, G. You, and W. Zhou, “A class of fast fixed-time synchronization control for the delayed neural network," Journal of the Franklin Institute, vol. 355, no. 1, pp. 164-176, 2018.

[47] Y. Wan, J. Cao, G. Wen, and W. Yu, "Robust fixed-time synchronization of delayed Cohen-Grossberg neural networks," Neural Networks, vol. 73, pp. 86-94, 2016.

[48] X. W. Liu and T. P. Chen, "Fixed-time cluster synchronization for complex networks via pinning control," 2015, https:// arxiv.org/abs/1509.03350.

[49] X. Yang, J. Lam, D. W. C. Ho, and Z. Feng, "Fixed-time synchronization of complex networks with impulsive effects via nonchattering control," IEEE Transactions on Automatic Control, vol. 62, no. 11, pp. 5511-5521, 2017.

[50] D. Meng, "Neural networks adaptive synchronization for four-dimension energy resource system with unknown dead zones," Neurocomputing, vol. 151, pp. 1495-1499, 2015.

[51] W. He and J. Cao, "Adaptive synchronization of a class of chaotic neural networks with known or unknown parameters," Physics Letters A, vol. 372, no. 4, pp. 408-416, 2008.

[52] Y. Xu, W. Zhou, J. A. Fang, and H. Lu, "Structure identification and adaptive synchronization of uncertain general complex dynamical networks," Physics Letters A, vol. 374, no. 2, pp. 272-278, 2009.

[53] D. Zhang, J. Mei, and P. Miao, "Global finite-time synchronization of different dimensional chaotic systems," Applied Mathematical Modelling, vol. 48, pp. 303-315, 2017.

[54] J. Zhao, Y. Wu, and Y. Wang, "Generalized finite-time synchronization between coupled chaotic systems of different orders with unknown parameters," Nonlinear Dynamics, vol. 74, no. 3, pp. 479-485, 2013.

[55] I. Ahmad, M. Shafiq, A. B. Saaban, A. B. Ibrahim, and M. Shahzad, "Robust finite-time global synchronization of chaotic systems with different orders," Optik, vol. 127, no. 19, pp. 8172-8185, 2016.

[56] L. Zhang, Y. Lei, Y. Wang, and H. Chen, "Generalized outer synchronization between non-dissipatively coupled complex networks with different-dimensional nodes," Applied Mathematical Modelling, vol. 55, pp. 248-261, 2018.

[57] Y.-L. Huang, W.-Z. Chen, and J.-M. Wang, "Finite-time passivity of delayed multi-weighted complex dynamical networks with different dimensional nodes," Neurocomputing, vol. 312, pp. 74-89, 2018.

[58] J. Lu, Y. Guo, Y. Ji, and S. Fan, "Finite-time synchronization for different dimensional fractional-order complex dynamical networks," Chaos, Solitons \& Fractals, vol. 130, p. 109433, 2020.

[59] H. Liu, H. Wang, J. Cao, A. Alsaedi, and T. Hayat, "Composite learning adaptive sliding mode control of fractional-order nonlinear systems with actuator faults," Journal of the Franklin Institute, vol. 356, no. 16, pp. 9580-9599, 2019.

[60] H. Liu, Y. Pan, J. Cao, H. Wang, and Y. Zhou, "Adaptive neural network backstepping control of fractional-order nonlinear systems with actuator faults," IEEE Transactions on 
Neural Networks and Learning Systems, vol. 31, no. 12, pp. 5166-5177, 2020.

[61] G. H. Hardy, J. E. Littlewood, and G. Pólya, Inequalities, Cambridge University Press, London, U. K., 1951.

[62] Z. Zuo and L. Tie, "Distributed robust finite-time nonlinear consensus protocols for multi-agent systems," International Journal of Systems Science, vol. 47, no. 6, pp. 1366-1375, 2016. 